\title{
Tea polyphenol modified, photothermal responsive and ROS generative black phosphorus quantum dots as nanoplatforms for promoting MRSA infected wounds healing in diabetic rats
}

Shibo Xu, Linna Chang, Yanan Hu, Xingjun Zhao, Shuocheng Huang, Zhenhua Chen* ${ }^{*}$, Xiuli Ren ${ }^{*}$ and Xifan Mei*

\begin{abstract}
Background: Healing of MRSA (methicillin-resistant Staphylococcus aureus) infected deep burn wounds (MIDBW) in diabetic patients remains an obstacle but is a cutting-edge research problem in clinical science. Surgical debridement and continuous antibiotic use remain the primary clinical treatment for MIDBW. However, suboptimal pharmacokinetics and high doses of antibiotics often cause serious side effects such as fatal complications of drug-resistant bacterial infections. MRSA, which causes wound infection, is currently a bacterium of concern in diabetic wound healing. In more severe cases, it can even lead to amputation of the patient's limb. The development of bioactive nanomaterials that can promote infected wound healing is significant.

Results: The present work proposed a strategy of using EGCG (Epigallocatechin gallate) modified black phosphorus quantum dots (BPQDs) as therapeutic nanoplatforms for MIDBW to achieve the synergistic functions of NIR (nearinfrared)-response, ROS-generation, sterilization, and promoting wound healing. The electron spin resonance results revealed that EGCG-BPQDs@H had a more vital photocatalytic ability to produce singlet oxygen than BPQDs@H. The inhibition results indicated an effective bactericidal rate of $88.6 \%$ against MRSA. Molecular biology analysis demonstrated that EGCG-BPQDs significantly upregulated CD31 nearly fourfold and basic fibroblast growth factor (bFGF) nearly twofold, which were beneficial for promoting the proliferation of vascular endothelial cells and skin epidermal cells. Under NIR irradiation, EGCG-BPQDs hydrogel (EGCG-BPQDs@H) treated MIDBW area could rapidly raise temperature up to $55^{\circ} \mathrm{C}$ for sterilization. The MIBDW closure rate of rats after 21 days of treatment was $92.4 \%$, much better than that of $61.1 \%$ of the control group. The engineered EGCG-BPQDs@H were found to promote MIDBW healing by triggering the PI3K/AKT and ERK1/2 signaling pathways, which could enhance cell proliferation and differentiation. In addition, intravenous circulation experiment showed good biocompatibility of EGCG-BPQDs@H. No significant damage to major organs was observed in rats.
\end{abstract}

*Correspondence: zhchen561@yahoo.com; rxlrenxiuli@163.com; meixifan1971@163.com

Jinzhou Medical University, Jinzhou 121001, Liaoning, China

(c) The Author(s) 2021. Open Access This article is licensed under a Creative Commons Attribution 4.0 International License, which permits use, sharing, adaptation, distribution and reproduction in any medium or format, as long as you give appropriate credit to the original author(s) and the source, provide a link to the Creative Commons licence, and indicate if changes were made. The images or other third party material in this article are included in the article's Creative Commons licence, unless indicated otherwise in a credit line to the material. If material is not included in the article's Creative Commons licence and your intended use is not permitted by statutory regulation or exceeds the permitted use, you will need to obtain permission directly from the copyright holder. To view a copy of this licence, visit http://creativecommons.org/licenses/by/4.0/. The Creative Commons Public Domain Dedication waiver (http://creativecommons.org/publicdomain/zero/1.0/) applies to the data made available in this article, unless otherwise stated in a credit line to the data. 
Conclusions: The obtained results demonstrated that EGCG-BPQDs@H achieved the synergistic functions of photocatalytic property, photothermal effects and promoted wound healing, and are promising multifunctional nanoplatforms for MIDBW healing in diabetics.

Keywords: Multifunctional nanoplatforms, Black phosphorus quantum dots, Diabetic deep-burn wound healing, Photocatalytic, Photothermal

\section{Graphical Abstract}

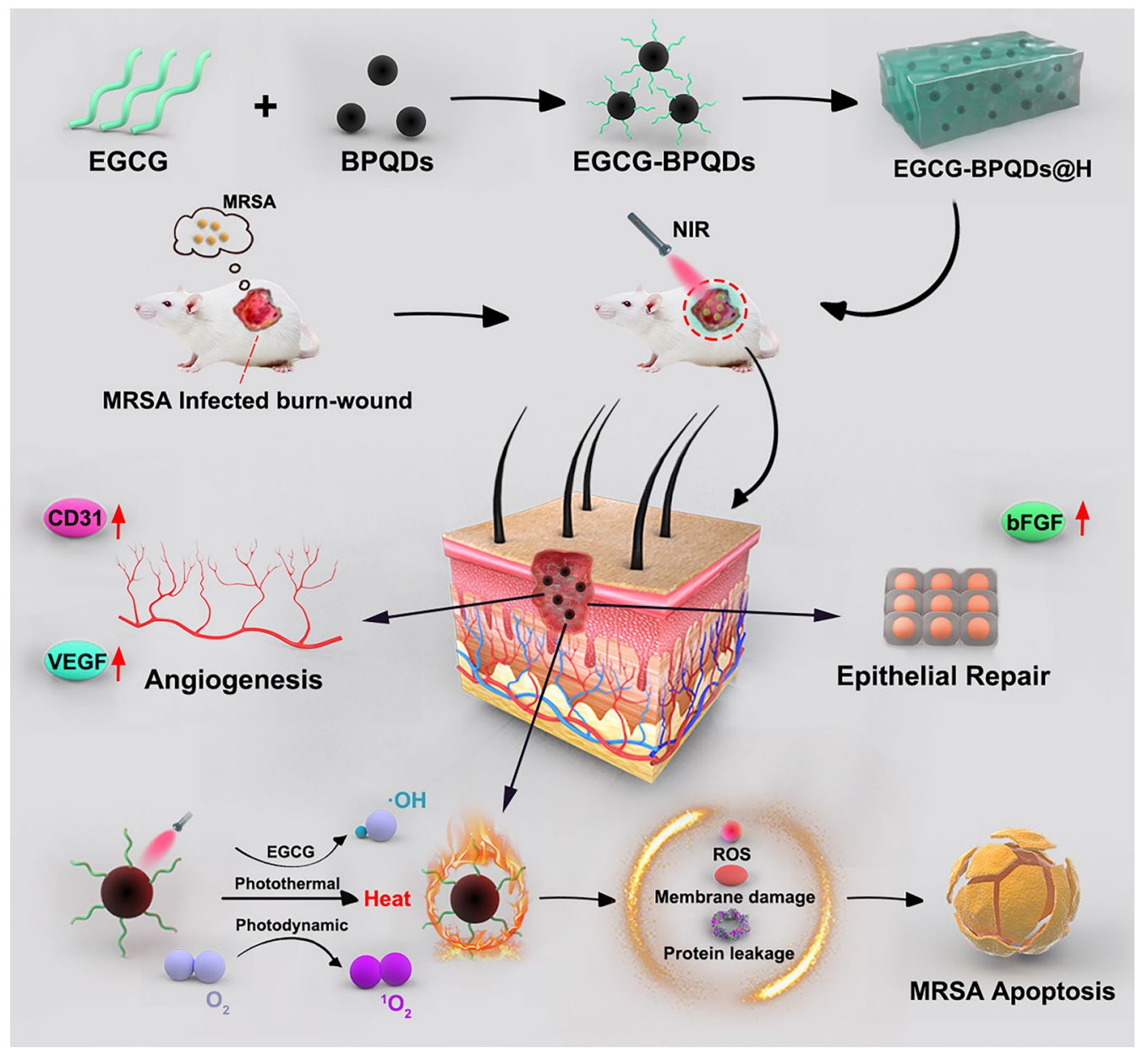

\section{Introduction}

MRSA infected deep-burn wound in diabetic patients is a fatal threat to human health and the frontier in clinical medicine [1]. Surgical debridement and continuous use of antibiotics are still the primary clinical treatment for MIDBW [2, 3]. However, suboptimal pharmacokinetics and large doses of antibiotics usually cause severe side effects, like deadly complications of resistant bacteria infection [4]. MRSA, which causes wound infections, is currently a major obstacle to the healing of diabetic wounds. It can even cause amputation of the patient's limb [5, 6]. Even worse, the development of new antibiotics has not been realized for a long time worldwide [7].
Therefore, besides surgical debridement and continuous use of antibiotics, multifunctional collaborative therapy nanoplatform attracts MIDBW in diabetic patients. To date, various nanomaterials have been currently available in diabetic-infected wound healing, such as metalorganic frameworks [8, 9], dopamine [10-13], peptides [14], metal oxides, and nano silver $[15,16]$. These excellent pioneered works suggest that multifunctional nanoplatforms with advantages of strong antibacterial and effective promoting of wound healing function are attractive, promising strategies for treating infected wounds.

Among these numerous antibacterial materials, black phosphorus (BP) is attractive because of its broad 


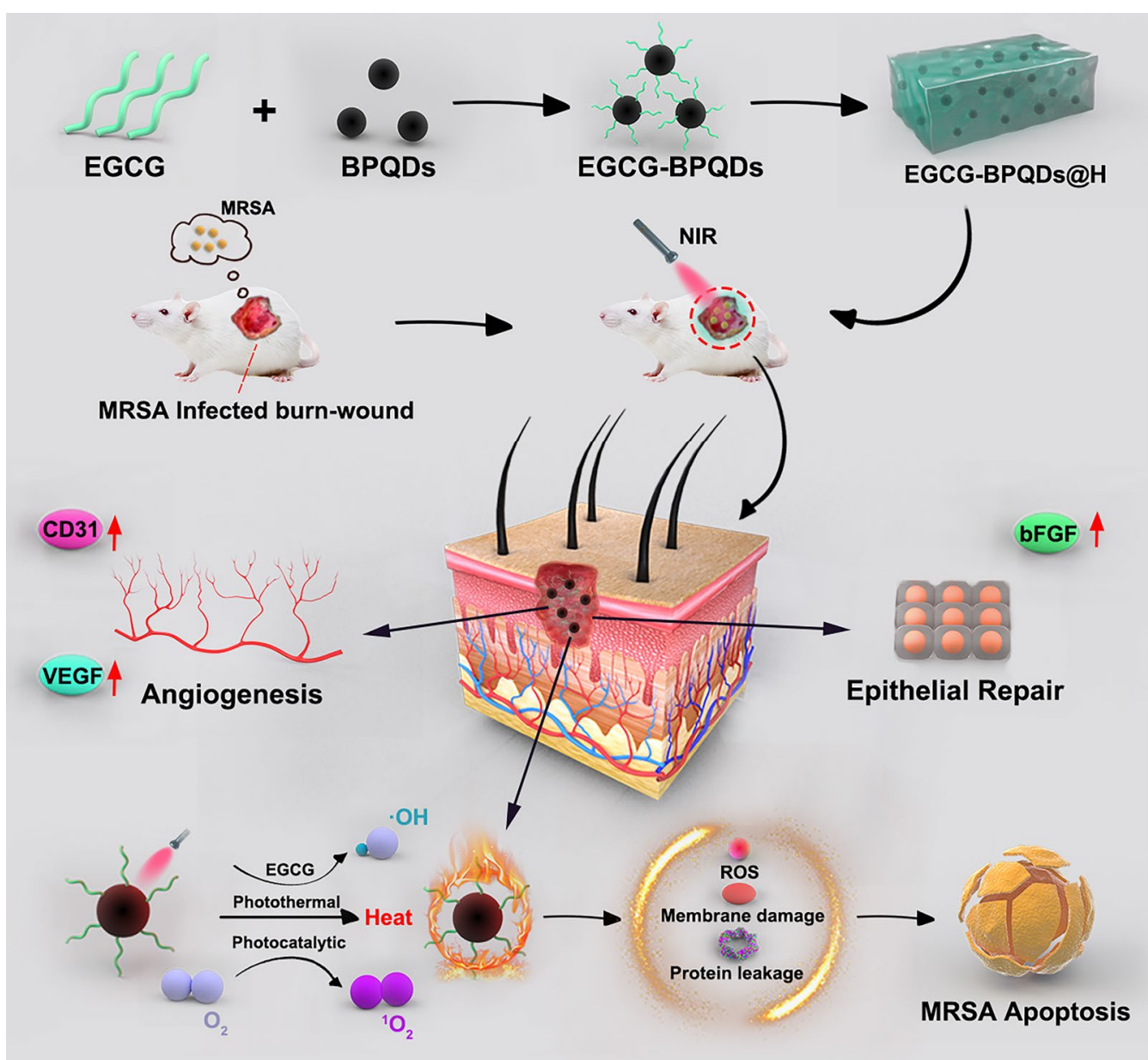

Scheme 1. The schematic illustration of the synthesis of EGCG-BPQDs@H nanocomposite and the process of sterilization and stimulation of cell behaviors that can promote regenerative activities of the skin cells and actively participate in epidermal regeneration, and accelerate the healing in diabetic infected wounds

optical absorption and striking charge carrier mobility, exhibiting outstanding singlet oxygen $\left({ }^{1} \mathrm{O}_{2}\right)$ activation and the high-temperature generation under NIR irradiation [17, 18]. Recently, photocatalytic properties and photothermal activity have been intensively investigated in biomedical applications [19], especially in the field of anti-infection wound treatments by their virtues of minimally invasive, effective sterilization, and easy operation [20-22]. These reported excellent works disclosed the attractive prospect of BP in infected wound healing. However, these documents mostly focused on BP nanosheets, while BPQDs have seldomly been researched, particularly in MIDBW. Therefore, the construction of a multifunctional BPQDs nanoplatform with collaborative properties of NIR response, ROS generation, sterilization, and promoted wound healing is a challengeable but attractive strategy for MIDBW therapy.
Herein, in order to achieve a multifunctional BPQDs nanoplatform, we proposed a strategy of constructing EGCG-modified BPQDs. As illustrated in Scheme 1, firstly, EGCG molecules were modified on BPQDs by the interactions between -OH group on EGCG and P atoms from BPQDs, such as hydrogen bond and molecule interactions. Previous works have proved the excellent biological function of tea polyphenol, especially EGCG [23-26]. Then, the obtained EGCG-BPQDs were loaded into hydrogels as a dual-purpose nano-agent to possess an antibacterial effect and simultaneously accelerate wound healing. Specifically, the engineered nanomaterials are expected to promote angiogenesis and epithelial regeneration by upregulating cellular CD31 and bFGF expression. Furthermore, under NIR irradiation, the nanomaterials will induce high levels of ROS production to eradicate antibiotic-resistant bacterial infections while 
generating local heat and accelerating microcirculatory blood flow. Our previous works and related reported works have explored hydrogels that can be used as excellent drug delivery systems [27]. Hydrogels encapsulated with EGCG-BPQDs will provide sustainable moisture and prolonged release of EGCG-BPQDs, which are beneficial for wound healing. Finally, the constructed hydrogels encapsulated EGCG-BPQDs are expected to achieve functional antibacterial, vascular regeneration, and accelerated epithelial regeneration.

\section{Materials and methods Materials}

Methicillin-resistant Staphylococcus aureus (MRSA) and Gram-negative bacteria Escherichia coli (E. coli) were purchased from Guangdong Microbial Culture Collection (China). Dulbecco's modified Eagle's medium (DMEM), 3-(4,5-Dimethylthiazol-2-yl)-2,5-diphenyl tetrazolium bromide (MTT), and fetal bovine serum (FBS) were purchased from Gibco (USA). Human skin keratinocytes cells (HaCat) and human umbilical vein endothelial cells (HUVEC) were obtained from American type culture collection (ATCC). The 2,2'-bis(anthracene9,10-diylbis(methylene))-dimalonic acid (ABDA) was supplied from Shanghai Civi Chemical Technology Co., Ltd. 1-methyl-2-pyrrolidone (NMP) was purchased from Aladdin. The primary antibodies to CD31, VEGF, bFGF, Tubulin were obtained from Cell Signaling Technology (USA). We used deionized water throughout the experiment.

\section{Characterization}

The component of EGCG-BPQD@H was identified by Fourier transform infrared spectroscopy (FTIR, SHIMADZU, Kyoto, Japan) with the $\mathrm{KBr}$ disk method. Relevant fluorescence data were acquired with a fluorescence photometer (F97PRO, Shanghai, China). UV-vis data were measured by UV-vis spectrophotometer (PerkinElmer Lambda 605S UV-vis spectrometer) at room temperature. The sample's crystal structure was tested by $\mathrm{X}$-ray diffraction (XRD, Shimadzu, Kyoto, Japan) and $\mathrm{Cu}$ $\mathrm{K}$ radiation. We also used dynamic laser scattering (DLS, Malvern, Nano ZS90, Worcestershire, UK) to test the sample's particle size. The sample's appearance characteristics were displayed through a transmission electron microscope (TEM, JEM-1200EX, Tokyo, Japan). Infrared thermal imager and NIR laser were purchased from Dongguan Bufan Electronics Co., Ltd., China. Confocal laser scanning microscopy (CLSM, Leica TSCSP5 confocal unit) was used to observe the expression of related proteins in cells.

\section{Therapy of burn wounds infected with MRSA in diabetic rats}

All animal experiments were carried out according to the Guidelines for Care and Use of Laboratory Animals of Jinzhou Medical University. Male Sprague-Dawley rats, weighing $190 \pm 20 \mathrm{~g}$, were obtained from Vital River Company (Beijing, China). After one week of acclimatization at the animal holding unit, the rats were ready for the following experiments. The diabetic animal model was established with streptozotocin (STZ; St. Louis Sigma, Missouri; $80 \mathrm{mg} / \mathrm{kg}$ ), and rats were injected intraperitoneally with STZ (0.1 M pH 4.5 citrate buffer formulation) once daily for four days. Rats with blood glucose levels greater than $300 \mathrm{mg} / \mathrm{dL}$ were selected for the test. Briefly, the dorsal hair was shaved and decontaminated after anesthetization, and then the electric device heated at $120^{\circ} \mathrm{C}$ was in contact with the shaved dorsal skin for $12 \mathrm{~s}$ to cause a diameter of $\sim 13 \mathrm{~mm}$ deep partial-thickness burn injury. Then the burn area was injected $100 \mu \mathrm{L}$ of MRSA suspension and was diluted to $2.0 \times 10^{7} \mathrm{CFU} /$ $\mathrm{mL}$ to build an experimental model of infection. After continuous infection for three days, the prepared hydrogel, BPQDs@H, EGCG@H, and EGCG-BPQDS@H were applied to the wound once daily directly. Meanwhile, the infected wound area was photographed at the same height, and a vernier caliper measured the size of wounds. The control+NIR, BPQDs@H+NIR, EGCG@H+NIR, and EGCG-BPQDS@H+NIR groups were treated with $808 \mathrm{~nm}$ laser $\left(2.5 \mathrm{~W} / \mathrm{cm}^{2}, 5 \mathrm{~min}\right)$, and the temperate changes of wounds were monitored via thermographic pictures captured by the infrared thermal imaging system. Then bodyweight of rats was registered every time. The burn wounds tissues and systemic organs were harvested for protein extraction and histological analyses.

\section{In vitro antibacterial activity analyses}

After activation and incubation, MRSA and E. coli were cultured at $37^{\circ} \mathrm{C}$ in a fresh liquid LB medium. The MRSA and $E$. coli $\left(1.0 \times 10^{8} \mathrm{CFU} / \mathrm{mL}\right)$ were briefly extended into the LB-Agar-Medium. After that, filter papers containing EGCG-BPQDs@H or other nanomaterials were spread on the MRSA and E. coli agar plates as a method of antibacterial activity evaluation. Each group's diameters of the bacteriostatic ring were measured after incubation for $24 \mathrm{~h}$. We also used MRSA and E. coli to estimate the synergetic antibacterial effects of EGCG-BPQDs@H plus NIR laser irradiation. Logarithmic growth phase bacteria were incubated in LB medium and then mixed with each group of materials in a $1.5 \mathrm{~mL}$ Solarbio tube containing $100 \mu \mathrm{L}$ of normal saline. For all groups, the bacteria were illuminated with/without NIR laser $\left(808 \mathrm{~nm}, 2.5 \mathrm{~W} / \mathrm{cm}^{2}\right)$ 
for $10 \mathrm{~min}$ and were incubated for another $24 \mathrm{~h}$ in the LB-Agar-Medium for CFU. In addition, to observe the antibacterial effect of different groups directly, bacterial morphology was examined by scanning electron microscope (SEM).

\section{Calcein-AM/PI staining}

The antibacterial effect was measured by the double fluorescent dye method. Specifically, bacteria were treated with hydrogel, BPQDs@H, EGCG@H, or EGCG$\mathrm{BPQDs} @ \mathrm{H}$ for $3 \mathrm{~h}$ at $37^{\circ} \mathrm{C}$. The bacteria were irradiated with/without NIR laser $\left(2.5 \mathrm{~W} / \mathrm{cm}^{2}, 10 \mathrm{~min}\right)$. Bacteria were incubated with calcein-AM and PI (Solarbio, China) after centrifuging at $5000 \mathrm{rpm}$ for $5 \mathrm{~min}$. Then, the bacteria were washed with phosphate-buffered saline (PBS) and placed on glass slides. The bacteria with different treatments were presented under a fluorescence microscope (Leica DM4000B, Germany). The red-fluorescent nucleic acid dye PI, which can penetrate the damaged cell wall, was used to mark dead bacteria. In contrast, the Calcein-AM that penetrated the living cell membrane was cleaved by the intracellular esterase to form Calcein, which emitted strong green fluorescence and remained in the live bacteria.

\section{Photocatalytic property of EGCG-BPQDs@H}

The ABDA probes were applied to detect singlet oxygen generation and time-varying changes. The fluctuation of ABDA probes was recorded to reflect the generation of ROS. We added EGCG-BPQDs@H into the $5 \mathrm{mM}$ ABDA solution and then irradiated it under the near-infrared laser. The test was performed every $5 \mathrm{~min}$. In addition, the single linear state of oxygen produced is detected by electron spin resonance spectroscopy.

The intracellular ROS level of bacteria was measured by fluorescent probe, 2',7'-dichlorodihydrofluorescein diacetate (DCFH-DA, Beyotime, China), which could be deacetylated and oxidized to fluorescent products. The MRSA, after various treatments, was fixed with $4 \%$ paraformaldehyde and then incubated with DCFH-DA probe for $30 \mathrm{~min}$ in the dark. The fluorescence imaging was immediately recorded with Leica DM4000B microscope. The fluorescent intensity was measured via ImageJ and Fiji12 plugin to quantify the total ROS level via EGCG-BPQDs@H.

\section{Determination of protein leakage}

The leakage of protein through the membrane of MRSA was observed after exposing different samples. NIRtreated groups were irradiated with $808 \mathrm{~nm}$ NIR light for $20 \mathrm{~min}$ at a power density of $2.5 \mathrm{~W} / \mathrm{cm}^{2}$. Subsequently, the bacterial suspension supernatant was collected by centrifugation at 12,000 rm for $5 \mathrm{~min}$. Finally, the supernatant liquid was immediately withdrawn and seeded into a 96-well plate. The protein intensities of different groups were determined by the microplate reader at $562 \mathrm{~nm}$ through the BCA assay kit (Beyotime, China) to determine the relative protein leakage of each sample.

\section{Biofilm formation assay}

MRSA biofilm model was employed to assess the antibiofilm ability of EGCG-BPQDs@H antibacterial nanoplat-

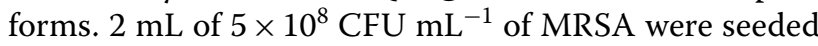
into a 24-well plate and incubated for $48 \mathrm{~h}$ to establish biofilm. After different treatments, crystal violet (CV) staining was utilized to evaluate the antibiofilm effect. MRSA biofilms were washed with gentle running deionized water to remove the unbound dye. The bound CV was dissolved using absolute ethanol. At last, a microplate reader was used to measure the absorbance of all samples at $590 \mathrm{~nm}$.

\section{Histology analyses}

In the infected burn wound healing experiment, individual wound tissues and major organs of the rats were harvested for histological analysis. The tissues were fixed in $4 \%$ formaldehyde and dehydrated through alcohol. The paraffin-embedded tissues were cut to a $5 \mu \mathrm{m}$ thickness and were stained with hematoxylin and eosin (H\&E) and Masson stain to evaluate the recovery of wound healing. Besides, the biocompatibility of nanomaterials in vivo as assessed by H\&E staining of main organs. The sections were captured with Leica DM4000B microscope.

\section{In vitro angiogenesis assay of HUVECs}

The angiogenesis ability of EGCG-BPQDs@H in vitro was assessed by endothelial tube-like formation as specified by the manufacturer's guidelines. HUVEC was used for this assay. The night before the experiment, pipette tips and well plates were placed in a $-20{ }^{\circ} \mathrm{C}$ freezer, and the Matrigel (BD Biosciences, USA) thawed at $4{ }^{\circ} \mathrm{C}$. First, $80 \mu \mathrm{L}$ Matrigel was incubated into a 96-well plate for $30 \mathrm{~min}$ at $37{ }^{\circ} \mathrm{C}$ to induce gelation. $1.0 \times 10^{4}$ HUVECs were first seeded on EGCG-BPQDS@H or BPQDs@H or EGCG@H with $100 \mu \mathrm{L}$ of FBS-free culture medium above solidified Matrigel for $6 \mathrm{~h}$. finally, the differentiation of HUVECs were observed through an inverted phase-contrast microscope (Leica DM4000B, Germany). The total length and number of nodes and segments were digitally imaged and quantified using the Angiogenesis Analyzer macro in ImageJ.

\section{In vitro HUVECs migration experiment}

Scratch test to assess the migration ability of HUVECs, which measures the expansion of HUVECs on margin. HUVECs were seeded at a density of $8 \times 10^{4} /$ well in a 
12-well plate with FBS-free medium to form a confluent monolayer. After incubation for $1 \mathrm{~d}$, a straight scratch was created by pipette tip, and the debris was gently washed twice with PBS. Then, each group was treated with their corresponding nanomaterials at $37{ }^{\circ} \mathrm{C}$ in the incubator. The cells were treated with $4 \%$ paraformaldehyde, incubated with $0.1 \%$ Triton X-100 (Sigma, USA), and then stained with 4',6-diamidino-2-phenylindole solution (DAPI, Invitrogen, USA). The difference between scratch wounds at $0 \mathrm{~h}$ and $24 \mathrm{~h}$ was captured with an inverted microscope, and the rate of cell migration was calculated as follows:

$$
\text { HUVECs migration }(\%)=\left(\mathrm{W}_{0 \mathrm{~h}}-\mathrm{W}_{12 \mathrm{~h}}\right) / \mathrm{W}_{0 \mathrm{~h}}, 1
$$

$\mathrm{W}_{0 \mathrm{~h}}$ was the initial wound area, and $\mathrm{W}_{12 \mathrm{~h}}$ was the wound area after $12 \mathrm{~h}$ of incubation.

\section{Cell viability assay}

The MTT assay was used to determine the proliferation of HUVECs in leach liquor of each group material and normal medium at different times. Firstly, HUVECs (5000 cells/well) were incubated in 96-well plates for $24 \mathrm{~h}$. After starvation with fetal bovine serum (FBS)-free medium overnight, the culture medium was removed and replaced by the different groups' leach liquor, respectively. They were incubated, and the solution in each well was pipetted out at 6,12 , and $24 \mathrm{~h}$. Subsequently, $20 \mu \mathrm{L}$ of MTT solution ( $5 \mathrm{mg} / \mathrm{mL}$ in FBS) was added to each well for $4 \mathrm{~h}$ at each time. Then, the supernatant was removed, and $150 \mu \mathrm{L}$ dimethyl sulfoxide (DMSO) was added to each well and incubated for $15 \mathrm{~min}$ in a dark place. Finally, quantitative detection was performed on a microplate reader at $490 \mathrm{~nm}$. In order to better mimic the microenvironment of bacterial infections in vivo, the cells were pretreated with $1 \mu \mathrm{g} / \mathrm{mL}$ lipopolysaccharide (LPS) followed by different nanomaterials treatments for $24 \mathrm{~h}$, and cells treated with LPS alone were used as a positive control.

\section{Western blot analysis}

Cells or tissues around the wound were collected and homogenized with RIPA (EnoGene, China) buffer to collect supernatants. Equal amounts of proteins $(15 \mu \mathrm{g})$ separated on $10 \%$ polyacrylamide gels and transferred on PVDF membrane, which was blocked with $5 \%$ skim milk for $2 \mathrm{~h}$. Then, the membranes were incubated with CD31, VEGF, bFGF, Tubulin antibodies followed by corresponding second antibodies for $2 \mathrm{~h}$ at indoor temperature. Immune reactivity was detected with a super signal ultrachemiluminescent reagent (Pierce Chemical, Rockford, IL, USA). Images were captured by Alpha Innotech Photo Documentation System (Alpha Innotech, Hayward, CA,
USA). Quantification of the protein expression was performed by ImageJ software.

\section{Immunofluorescence double labeling method}

The leach liquor of each group was added to the well plate. In each group, HUVECs and HaCaTs were washed 3 times with PBS and treated with 4\% PFA for $40 \mathrm{~min}$. After incubated with $0.1 \%$ Triton X-100, cells were washed 3 times and blocked with $5 \%$ goat serum for $2 \mathrm{~h}$. Cells were also incubated with primary anti-bFGF (1:1000, CST, USA); anti-Tubulin $(1 ; 1000$, CST, USA); anti-CD31 (1;500, Abcam, UK); anti-VEGF (1;500, Abcam, UK) overnight at $4{ }^{\circ} \mathrm{C}$. Subsequently, they were washed 3 times and incubated with Alexa Fluor 488 goat anti-rabbit IgG or Alexa Fluor 594 goat anti-mouse IgG (1;500, Thermo, USA) for $2 \mathrm{~h}$. Nuclei were stained with DAPI for 15 min. After washing, confocal laser scanning microscopy was used for other cells characterization.

\section{Statistical analysis}

All independent experiments were performed in triplicate in the corresponding condition. All Graphs were rendered using GraphPad Prism software, version 6 . One-way analysis of variance (ANOVA) with post hoc Tukey multiple comparison tests was used to analyze statistical significance. Differences between different groups at ${ }^{*} \mathrm{P}<0.05,{ }^{* *} \mathrm{P}<0.01,{ }^{* * *} \mathrm{P}<0.001$ were considered as statistically significant.

\section{Results and discussion}

\section{Preparation and characterization of EGCG-BPQDs@H}

The black phosphorus quantum dots were obtained by the liquid-phase ultrasonic stripping method, and $500 \mathrm{mg}$ of black phosphorus powder was put into $50 \mathrm{~mL}$ of N-methyl pyrrolidone (NMP), mixed, and ground in a mortar. The mixture was sonicated in an ice bath for $4 \mathrm{~h}$, and then centrifuged at a speed of 12,000 r/ min for $20 \mathrm{~min}$. The supernatant containing BPQDs was decanted. We then carried out a series of related experiments to verify the successful preparation of the composite. Firstly, we used HRTEM to observe the morphological characteristics of EGCG-BPQDs. As shown in Fig. 1A, we can see homogeneous EGCG-BPQD nanoparticles. The insert in Fig. $1 \mathrm{~A}$ is the image of one single magnified BPQD. The lattice fringes of the BPQD could be discriminated. The DLS indicated that the size of BPQDs is about $3 \mathrm{~nm}$ (Fig. 1B).

The results of FTIR spectra showed that the absorption peak at $1718 \mathrm{~cm}^{-1}$ and the broad absorption peak at $1400-1600 \mathrm{~cm}^{-1}$ were characteristic absorption peaks of $-\mathrm{C}=\mathrm{O}$ and benzene of EGCG, $1100 \mathrm{~cm}^{-1}$, and $1250 \mathrm{~cm}^{-1}$ were $\mathrm{P}=\mathrm{O}$ stretching vibration peak. It is proved that the prepared EGCG-BPQD@H already contains EGCG 


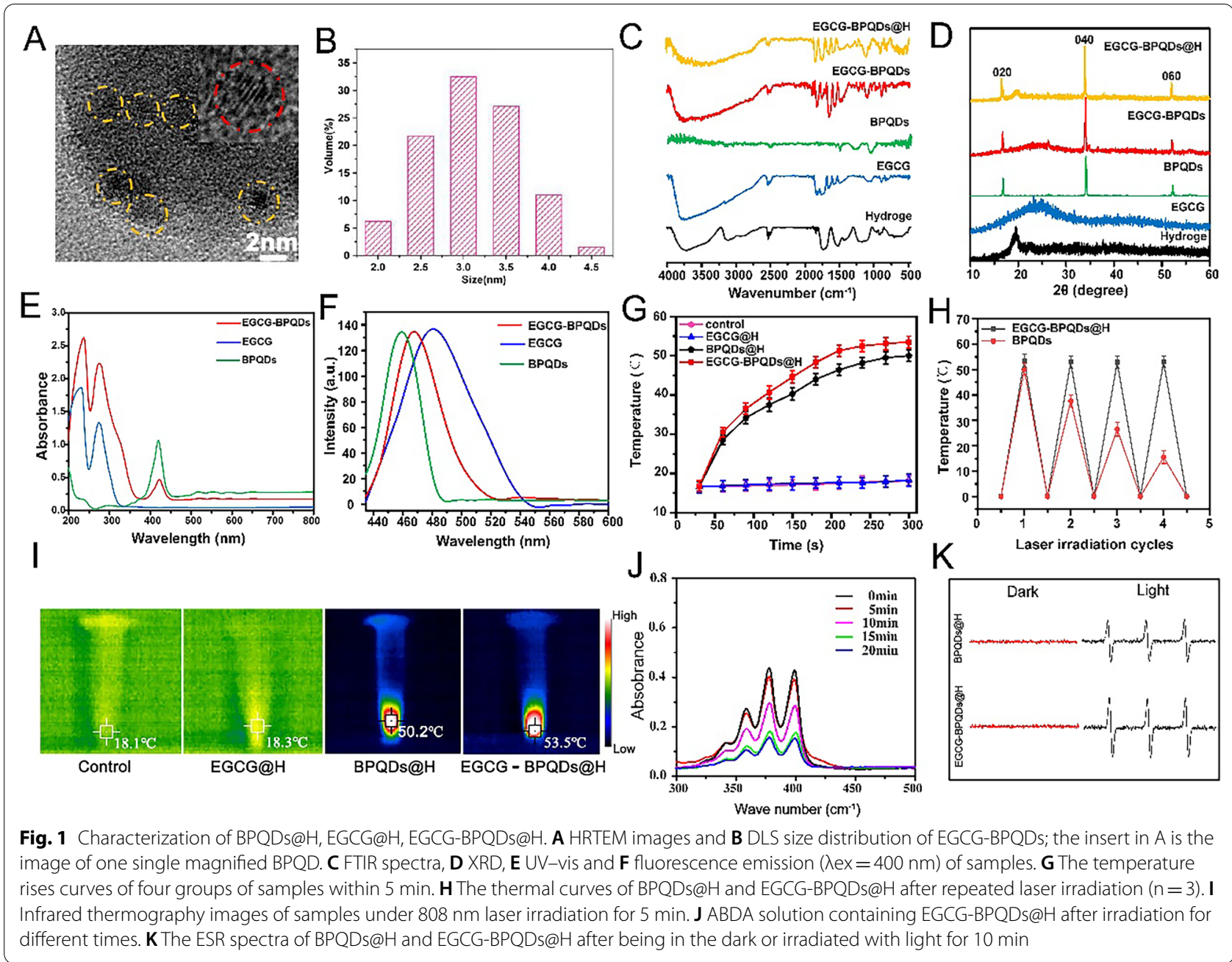

and BPQD (Fig. 1C). In addition, the synthetic hydrogels were tested by FTIR (Additional file 1: Fig. S1). We tested the crystal form of the synthesized product by XRD. In Fig. 1D, the diffraction peaks at $\sim 16^{\circ}, 34^{\circ}$, and $52^{\circ}$ corresponded well to the diffraction peaks of the (020), (040), and (060) crystal planes of BPQD, showing that BPQD still maintains a complete structure when it was fused to the hydrogel or bonded to EGCG (Fig. 1D). We demonstrated the successful preparation of EGCG-BPQDs by UV-vis spectroscopy. As shown in Fig. 1E. BPQDs exhibited a strong and sharp absorption peak at approximately $420 \mathrm{~nm}$, which indicated the successful synthesis of high-quality BPQDs [28]. Moreover, the characteristic absorption peaks of EGCG and BPQDs can be observed in EGCG-BPQDs, which again demonstrated that we have successfully synthesized EGCG-BPQDs. The fluorescence spectra demonstrated that the emission peaks of BPQDs and EGCG appear at $460 \mathrm{~nm}$ and $481 \mathrm{~nm}$, respectively, and the peak of EGCG-BPQDs@H appears at $467 \mathrm{~nm}$ (Fig. 1F). These results indicated that we have successfully prepared EGCG-BPQDs and well dispersed in hydrogels.

To verify the photothermal capability of EGCGBPQDs@H in photothermal therapy, we irradiated EGCG-BPQDs@H with a NIR laser $\left(2.5\right.$ W/ $\left./ \mathrm{cm}^{2}, 808 \mathrm{~nm}\right)$ for $5 \mathrm{~min}$ and recorded the temperature change with a thermal imager (Fig. 1G and I). As shown in Fig. 1H, the temperature of EGCG-BPQDs@H and BPQDs@H increased to $53.5{ }^{\circ} \mathrm{C}$ and $50.2{ }^{\circ} \mathrm{C}$, while the temperature of EGCG@H only changed to $1.7{ }^{\circ} \mathrm{C}$, indicating that EGCGBPQDs@H have excellent photothermal performance while its maximum photothermal conversion efficiency was reaching 46.7\% (Additional file 1: Fig, S2). Finally, the stability of EGCG-BPQDs@H under cyclic light was also further explored. The samples were irradiated for the same duration and cycle (4 cycles, 5 min each cycle). The highest temperature that the samples could reach after each cycle was recorded using a thermal imager (Fig. 1H). At the second cycle, the maximum temperature of the BPQDs began to decrease rapidly, reaching a maximum 
temperature of just $15.5{ }^{\circ} \mathrm{C}$ by the fourth cycle. In contrast, maximum temperature of the EGCG-BPQDs@H remained near $52{ }^{\circ} \mathrm{C}$ and still maintained good photothermal properties. These results indicate that the hydrogels provided some protection to the BPQDs and reduced the degradation of the BPQDs.

The production of singlet oxygen is an important basis for assessing the effectiveness of the photocatalytic effect with photosensitizers. We evaluated the ability of EGCGBPQDs@H to produce singlet oxygen under NIR irradiation using ADBA solution. Under NIR conditions, the intensity of the absorption peak of the ABDA solution at $380 \mathrm{~nm}$ decreased sharply, indicating that the material produces a large amount of singlet oxygen (Fig. 1J). In contrast, the absorption spectra of the ABDA solution alone or the EGCG@H group did not change significantly under NIR irradiation (Additional file 1: Fig. S3A, B). To further validate the conclusions, electron spin resonance measurements were performed to verify the ability of the material to produce singly linear oxygen. After 5 min of irradiation, typical electron spin resonance spectra were observed (Fig. 1K). The relative intensity of the electron spin resonance produced by EGCG-BPQDs@H was significantly higher than BPQDs@H for the same recording time, indicating that EGCG-BPQDs@H had a stronger ability to produce singlet oxygen. In vitro release experiments with EGCG@H were performed using dialysis bags and demonstrated that free EGCG showed a faster release rate. In contrast, EGCG@H and EGCGBPQDs@H could control the slow and smooth release of EGCG (Additional file 1: Fig. S3C). UV-vis indicated that more EGCG was released from EGCG-BPQDs@H under NIR irradiation, which contributed to our further modulation of drug release (Additional file 1: Fig. S3D).

\section{In vitro antibacterial properties of EGCG-BPQDs@H}

The agar spread assay was performed to evaluate the antimicrobial capacity of different nanomaterials. After $37^{\circ} \mathrm{C}$ constant temperature cultivation, treatment with hydrogel did not show antibacterial effect for the tested bacterial. On the contrary, antibacterial rings of tested bacteria were clearly observed after treatment with BPQDs@H, EGCG@H, or EGCG-BPQDs@H (Fig. 2A). The diameters of the EGCG-BPQDs@H group antibacterial zone towards $E$. coli and MRSA were $\sim 23 \mathrm{~mm}, \sim 21 \mathrm{~mm}$, respectively (Fig. $2 \mathrm{~B}$ and $\mathrm{C}$ ), demonstrating its obvious antibacterial activity. These results presented a stronger antibacterial effect after treatment with EGCGBPQDs@H, attributed to the synergistic relationship between EGCG and BPQDs. In addition, experiments demonstrated that BPQDs could cause bacterial toxicity in the absence of NIR. MRSA and E. coli cells from different treatments were diluted and spread on the agar plates, respectively. Next, the colony counts assay investigated the antibacterial effect of black phosphorous quantum dots (Fig. 2D and F). The results of the colony count assay without NIR were in harmony with the antibacterial ring. Nevertheless, the bacteria viability in BPQDs@H+ NIR treated groups was only 3.2\% for E. coli and $11.3 \%$ for MRSA, respectively (Fig. 2E and G).

These data also suggested the stronger antibacterial effect empowered by phototherapy. Anyhow, these results indicated that neither EGCG-BPQDs@H nor photothermal treatment could eliminate the selected bacteria. On the contrary, especially in E. coli, almost no bacterial colonies were detected after combinational treatment EGCG-BPQDs@H under NIR irradiation. That is to say, the EGCG-BPQDs@H+NIR could more efficiently suppress bacterial viability and inhibit the growth of E. coli and MRSA. Briefly, the local thermal heat produced by nanomaterials under NIR irradiation was able to destroy bacteria by deactivating enzymes and disrupting metabolism in bacteria. Moreover, the NIR radiation provoked the release of EGCG from EGCG-BPQDs@H, which increased the local EGCG concentration. The high EGCG content affected bacterial activity and led to bacterial death [29]. Therefore, cooperative treatment was a prominent strategy to eliminate bacteria.

\section{Evaluation of the integrity of bacteria}

The bacterial survival rate was further identified through fluorescence staining assays to evaluate the antibacterial effect of the cooperative treatment. Fluorescence pictures of live/dead staining showed that only intense green fluorescence and no visible red fluorescence were observed in bacteria after treatment with normal hydrogels or with NIR only. By contrast, the BPQDs@H, EGCG@H, EGCGBPQDs@H, BPQD@H+NIR, and EGCG@H+NIR treated bacterial emitted red fluorescence as well as green fluorescence, suggesting regional antibacterial efficacy was achieved (Fig. 3A). The strongest red fluorescence could be detected in the group in which the bacterial were treated with EGCG-BPQDs@H with NIR, indicating the potent antibacterial activity of the cooperative treatment (Fig. 3B). SEM was used to observe the cellular morphological changes of MRSA following the different treatments (Fig. 3C). As demonstrated in SEM pictures, it was found that bacterial remained.

typical spherical shape and intact smooth surface after treatment with normal hydrogel, NIR. In the case of the MRSA treated with BPQDs@H, EGCG@H, EGCG-BPQDs@H, BPQD@H+NIR, or EGCG@H+NIR, obvious disruption appeared on their original morphology, indicating their surface became slightly rough and fragmentary. In contrast, the structure of bacteria was distorted and displayed 


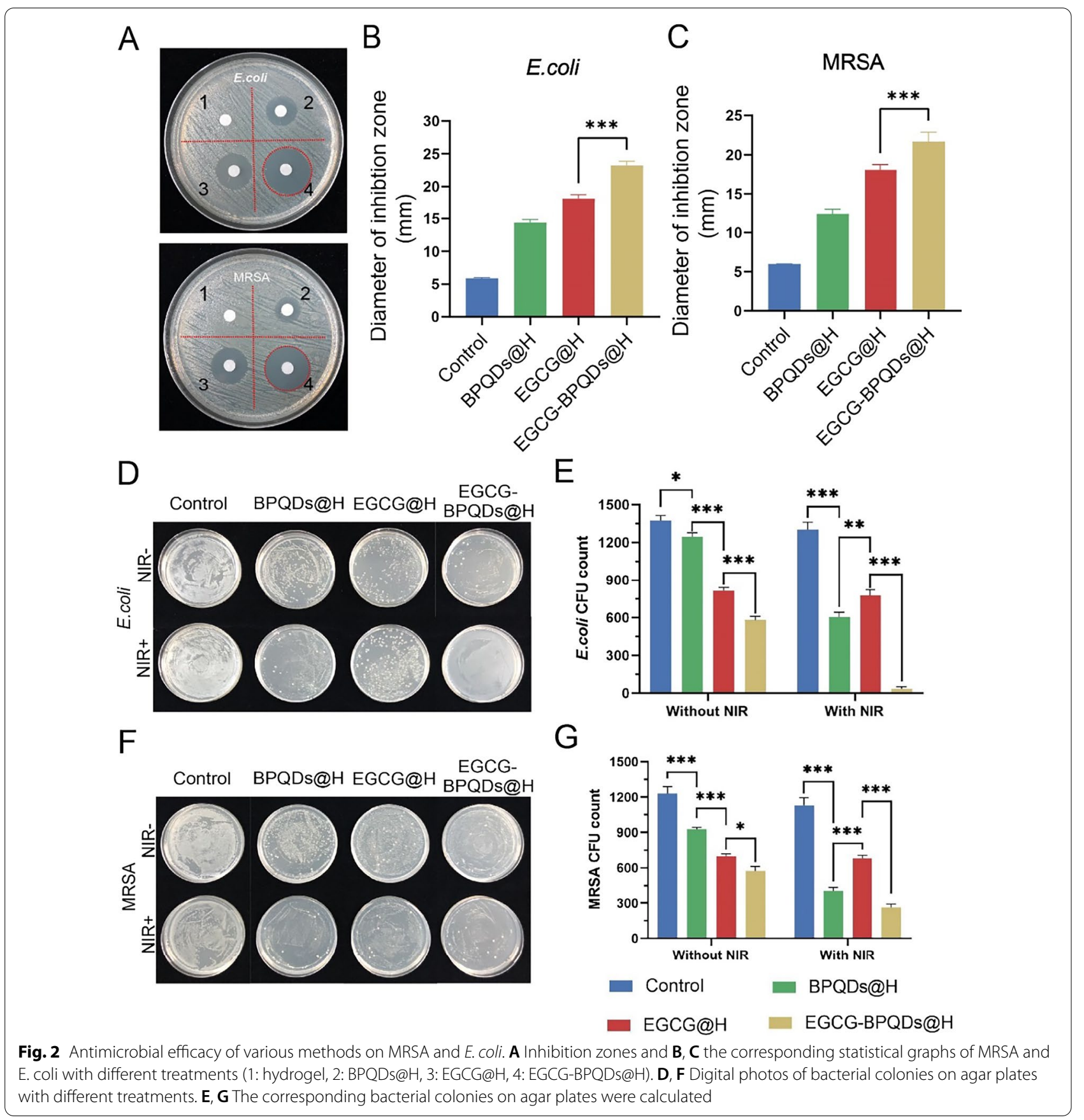

more serious damage after combining with EGCGBPQDs@H under NIR irradiation, implying the strongest antibacterial effect of the cooperative treatment. Besides the special photothermal property of BPQDs can convert near-infrared light into local high temperature to denature the enzymes in the bacteria, the phenolic hydroxyl of EGCG could bind to the phospholipid bilayer of bacteria and the amino and carboxyl groups in bacterial membrane proteins, which was reported to disrupt the integrity of the bacterial membrane [30].

\section{Antibacterial mechanism exploration in vitro}

In addition to the above results, the active oxygen generated under radiation was further examined to explore the antibacterial mechanism and antibacterial effect. DCFH-DA was used to monitor the generation of ROS 


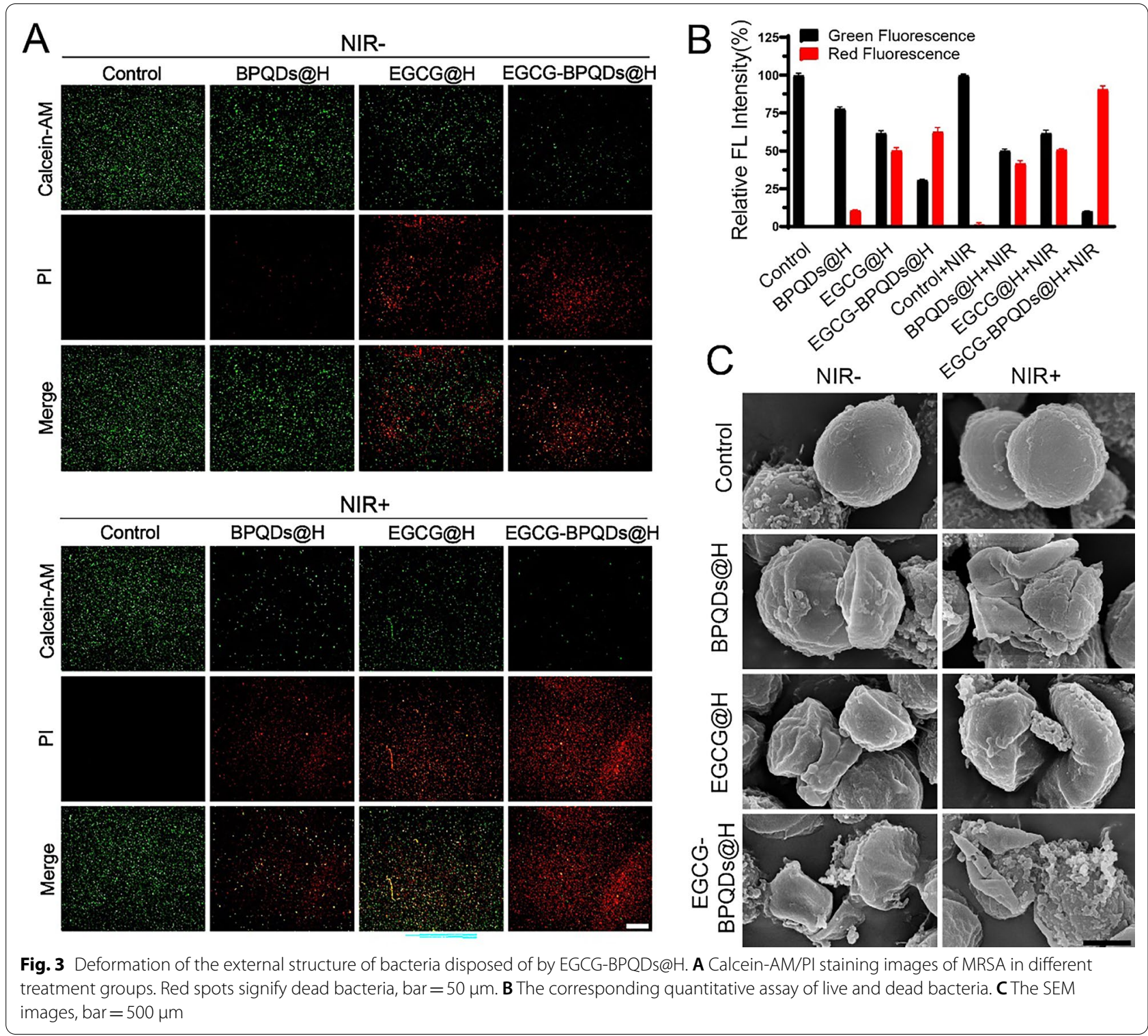

after different treatments on MRSA. As a potential photocatalyst, black phosphorous nanomaterials could generate numerous singlet oxygen $\left({ }^{1} \mathrm{O}_{2}\right)$, introducing reactive oxygen-dependent oxidative stress and membrane damage [31-34]. It was observed that little green fluorescence of the bacteria was detected after treatment with normal saline or NIR. Whereas the laser irradiation significantly enhanced ROS production in BPQDs@H and EGCG-BPQDs@H treated group. More importantly, a much higher level of ROS was generated in EGCG-BPQDs@H treated group, partly attributed to the spontaneous slow release of EGCG. Meanwhile, comparing with EGCG-BPQDs@H group,
ROS production was obviously upregulated in EGCGBPQDs@H+NIR group due to the EGCG release and high temperature (Fig. 4A and $\mathrm{C}$ ). Since the rupture of the membrane causes cytoplasm to leak, the degree of protein leakage can be used as a response to the antibacterial effect [35]. We further evaluated the leakage of intracellular protein after exposure to different groups. As shown in Additional file 1: Fig. S4, the leakage of proteins from the bacteria treated with the combination was significantly reduced. Nevertheless, in the other groups, only relatively weak changes were observed, indicating that EGCG-BPQDs@H caused severe disruption of the bacterial cell membrane under NIR irradiation. 

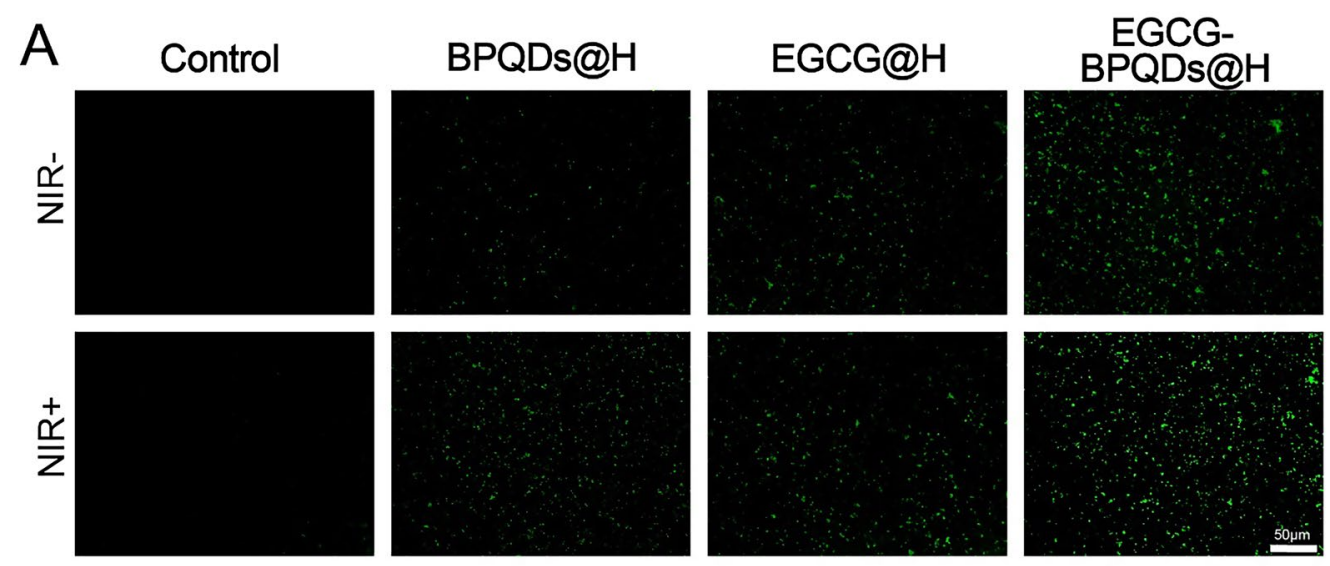

B

NIR-
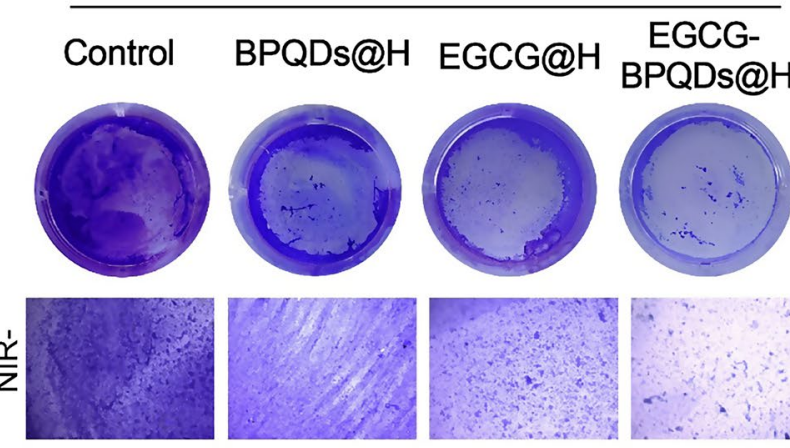

$\mathrm{NIR+}$

C
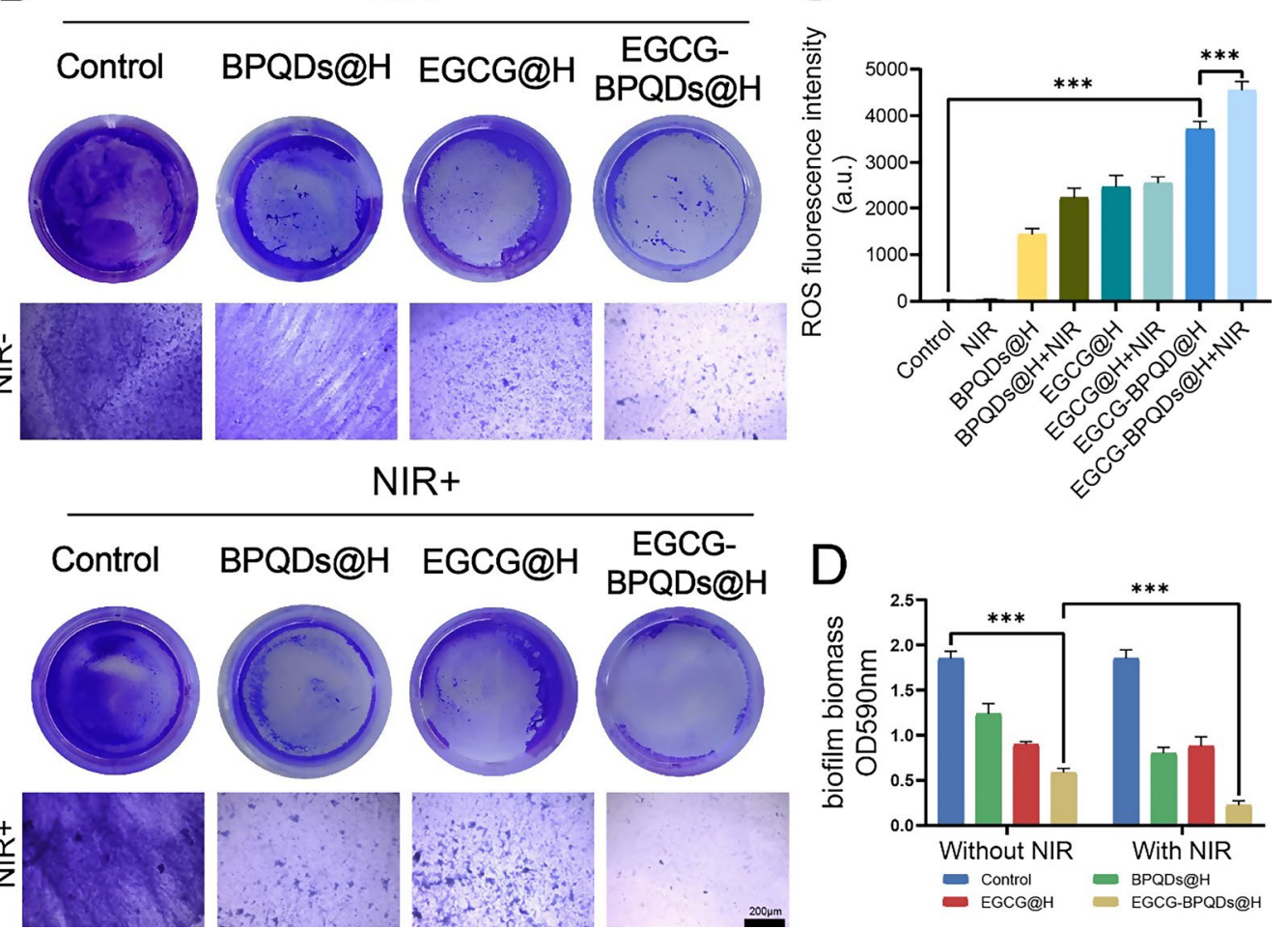

Fig. 4 The investigation of antibacterial mechanisms. A Fluorescent photographs of ROS level in MRSA with various treatments in vitro, stained by DCFH-DA, bar $=100 \mu \mathrm{m}$. B Crystalline violet-stained biofilm. E Images of MRSA biofilms stained with crystal violet, bar $=200 \mu \mathrm{m}$. C Consequential statistics of histograms indicating religious ROS activity levels. D Absorbance of biofilm in different groups

Biofilms have further weakened the therapeutic effect due to their resistance to antibiotic therapy [36]. Therefore, the antibacterial activity of EGCG-BPQDs@H against the MRSA biofilm was the further test. The darker the blue, the more biofilm stained by crystal violet. Limited radicalization was observable following normal saline or BPQDs@H treatment alone, and the radicalization activity was not related to NIR light (Fig. 4B). As shown in Fig. 4D, the corresponding absorbance and solution color of the group treated with EGCG-BPQDs@H+NIR were the least of all the treating sets, suggesting that the synergistic system of EGCG-BPQDs@H+NIR has an efficiency against biofilm. In brief, simply NIR radiation, EGCG@H, or the BPQDs@H cannot kill bacteria effectively. Under the NIR irradiation, the photothermal and photocatalytic properties of EGCG-BPQDs@H could be activated, together with increased release of EGCG, which 
exerted additional antibacterial effects to the bacteria system. EGCG-BPQDs@H excited by NIR laser present a higher bactericidal efficiency than EGCG-BPQDs@H. This enhancement might be due to the synergistic effect of photothermal and active oxygen sterilization. Comparison between EGCG-BPQDs@H and BPQDs@H also affirmed the positive efficacy of EGCG, and there was no significant change before and after NIR illumination. Accordingly, the NIR regulated photothermal, ROS and release promoting synergetic therapy could be used to completely combat multidrug-resistant bacterial infections. In addition, we obtained fluorescent images of bacterial biofilms after incubating the composite hydrogel-treated bacteria with FITC-Con A (Additional file 1: Fig. S5). The bacterial biofilm was significantly reduced after treatment with EGCG-BPQDs@H with NIR irradiation, demonstrating the good antibacterial properties of this hydrogel system for biofilms.

\section{Promoting migration and proliferation effects of EGCG-BPQDs@H in vitro}

It is commonly accepted that endothelial cell migration is critical in angiogenesis [37]. EGCG is considered to have a superior impact in accelerating wound healing by virtue of antibacterial, anti-inflammatory, and facilitating angiogenesis [38-40]. Therefore, the regenerative ability of EGCG-BPQDs@H was further investigated through HUVECs. Following the different treatments of cells scratch, the migration extent revealed a difference (Fig. 5A). Compared with other groups, significant cell migration was detected in EGCG-BPQDs@H group after $24 \mathrm{~h}$ incubation (Fig. 5B). The wound area in EGCGBPQDs@H group decreased dramatically by $52.8 \%$, while only $43.1 \%, 38.3 \%$, and $17.6 \%$ of the wound decreased in the EGCG@H,BPQDs@H, and control group. Cytocompatibility is key to wound healing as it is in direct contact with the wound tissue. Cell proliferation was also determined by MTT assay. The cell proliferation was sustainedly increased optical density (Fig. 5C). The proliferation of HUVECs after treatment of EGCG-BPQDs@H was significantly faster than other groups after 12- and 24-h incubation. The migration and proliferation among HUVECs indicated nanomaterials have good biocompatibility and the ability to improve wound healing, which could be attributed to the sustained release of EGCG, offering an appropriate microenvironment for distribution. Meanwhile, we evaluate the capacity of nanomaterials to promote epithelial by detecting the expression of basic fibroblast growth factor (bFGF) in $\mathrm{HaCaT}$ cells. It is widely accepted that bFGF.

is a kind of growth factor that takes a significant role in the therapy of skin trauma, neovascularization, and tissue regeneration [41]. Immunofluorescence observed on
CLSM revealing expression of bFGF protein in $\mathrm{HaCaTs}$ was much higher in EGCG-BPQDs@H treatment (Fig. 5D).

Through western blot (Fig. 5E) and relative western blot quantitative analysis (Fig. 5F), enhanced expression of bFGF was discovered in EGCG-BPQDs@H group. Although the bFGF protein levels of other signal groups were higher than the control group, both were lower than the EGCG@BOQDs@H group, indicating the synergistic properties of black phosphorus quantum dots and EGCG. Therefore, the experimental results confirmed that the nanomaterials could accelerate epithelial healing by promoting the expression of bFGF in epithelial cells.

\section{EGCG-BPQDs@H facilitated vascularization}

Angiogenesis is developing new capillaries to form the original microvasculature, which is a crucial stage in wound healing [42]. Total processes involved the proliferation, migration, alignment, and germination of endothelial cells and connections between cells, tubular structures, and lumina [43]. We conducted the Matrigel experiment to simulate the angiogenesis of endothelial cells on connective tissue membrane. As shown in Fig. 6A, HUVECs incubated on the Matrigel after different materials treatments formed tube networks and mesh-like circles to a different degree. The HUVECs treated with EGCG@H and EGCG-BPQDs@H exhibited more notable angiogenesis indexes. Furthermore, longer total lengths, enhanced number of nodes were observed in EGCG-BPQDs@H treatment (Fig. 6B and C). These results identified that EGCG-BPQDs@H served as an ideal extracellular matrix to facilitate endothelial cell tubule formation in vitro, which may accelerate endothelization and angiogenesis in impaired tissues.

To further identify our inference, we performed CLSM and western blot to examine the expression of the angiogenesis-associated proteins. It has been widely demonstrated that CD31 and VEGF can assess the level of angiogenesis. The highly expressed CD31 indicates the tight junctions between endothelial cells and is involved in the formation of blood vessels [44]. EGCG@H exhibited a modicum improvement in the expression of CD31. By contrast, EGCG-BPQDs@H further enhanced the expression level of CD31 (Fig. 6D). Moreover, the result of western blot acted by immunofluorescence staining (Fig. 6E). The expression of CD31 in the group with the treatment of EGCG@ BOQDs@H was remarkably improved compared to other groups (Fig. 6F). Undoubtedly, the expression of VEGF in EGCG-BPQDs@H treatment was higher than other groups. All these results suggested that we had proved once again that EGCG-BPQDs@H could ameliorate wound healing through promoting angiogenesis 
A
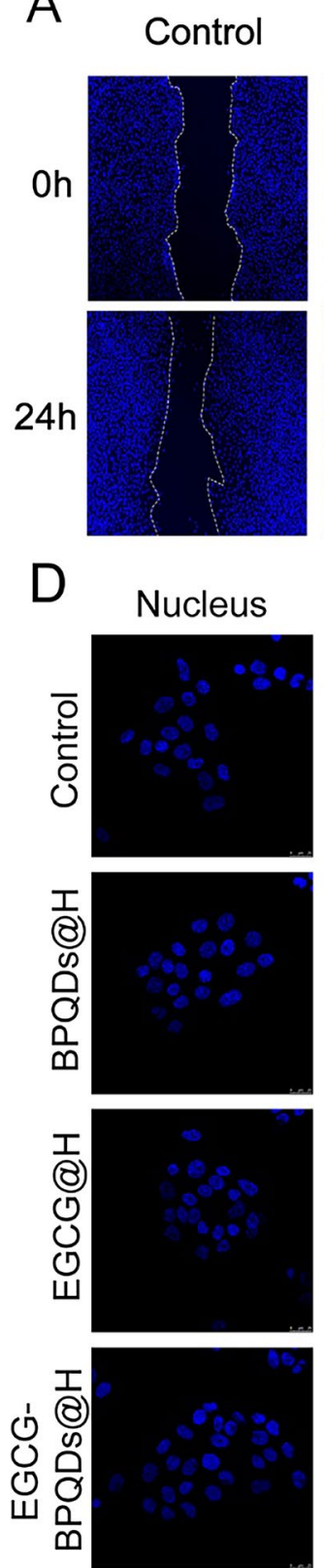

BPQDs@H
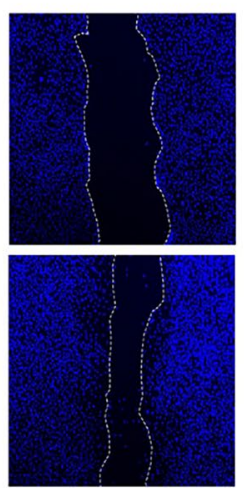

Tubulin
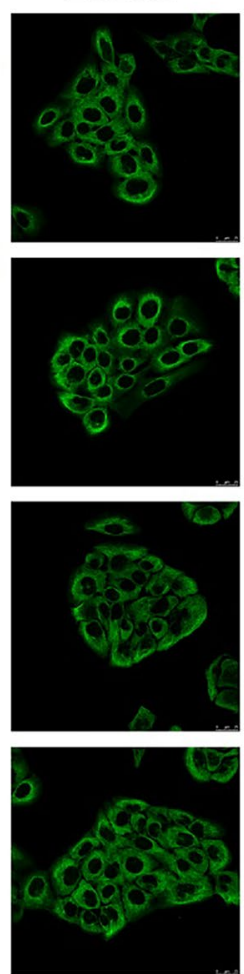
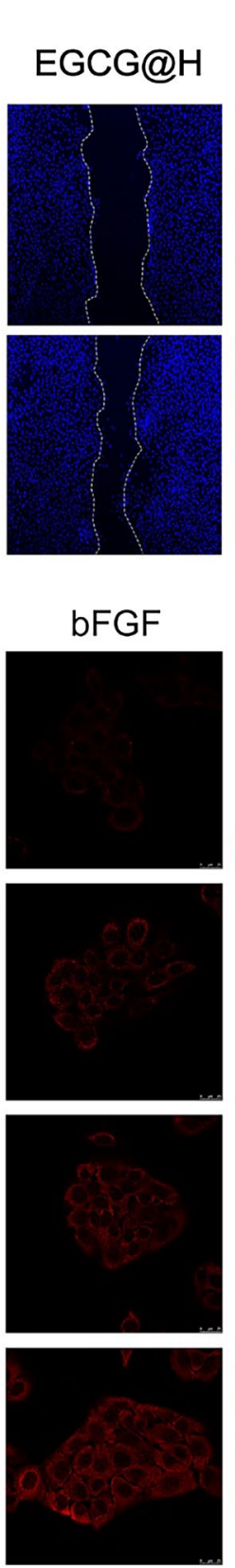
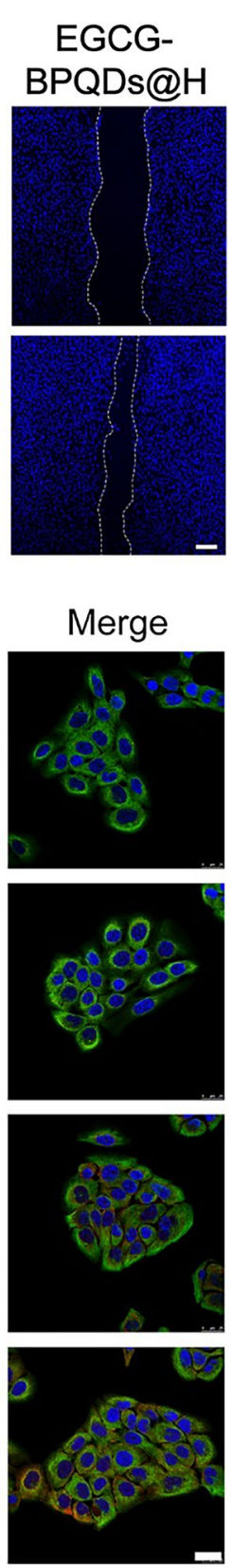

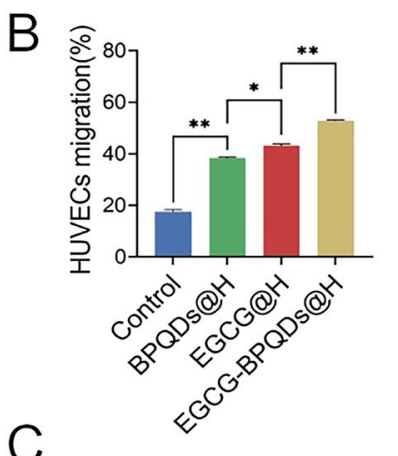

C

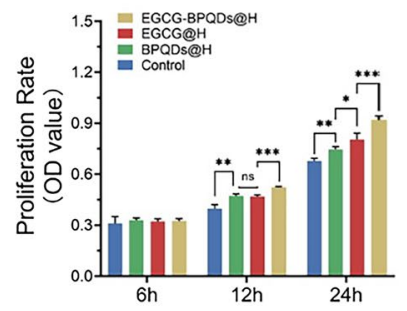

$E$

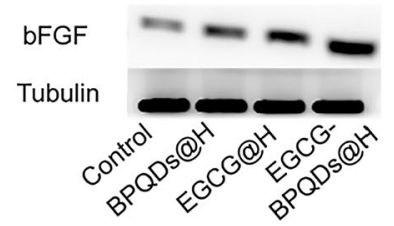

$\mathrm{F}$

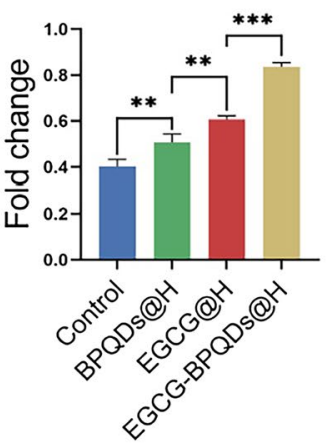

Fig. 5 Promoting migration and proliferation effects and potential mechanism. A Digital images of scratch wound assay in different treatments, bar $=100 \mu \mathrm{m}$. B Quantification of HUVEC migration. C MTT analysis in different groups. D CLSM images of the expression analysis of bFGF, bar $=25 \mu \mathrm{m}$. E The expression level of bFGF protein. $\mathbf{F}$ Quantification analysis of the bFGF protein. ${ }^{*} P<0.05,{ }^{* *} P<0.01,{ }^{* * *} P<0.001$

according to the relative protein expression. In combination with these findings, our study provides supporting evidence for using NIR-responsive, synergistic antimicrobial, sustained-release hydrogels of EGCGmodified BPQDs to promote MIDBW healing. To further investigate the healing effect in more detail, we carried out experiments in vivo.
In vivo assessment of burn-wound healing

To further explore the therapeutic benefits of EGCGBPQDs@H in burn-wound healing in vivo, the digital photographs of burn-wound showed male Sprague-Dawley diabetic rats infected burning wounds (Fig. 7A). The burn-wounds of diabetic rats treated with EGCGBPQDs@H with or without NIR revealed accelerated 


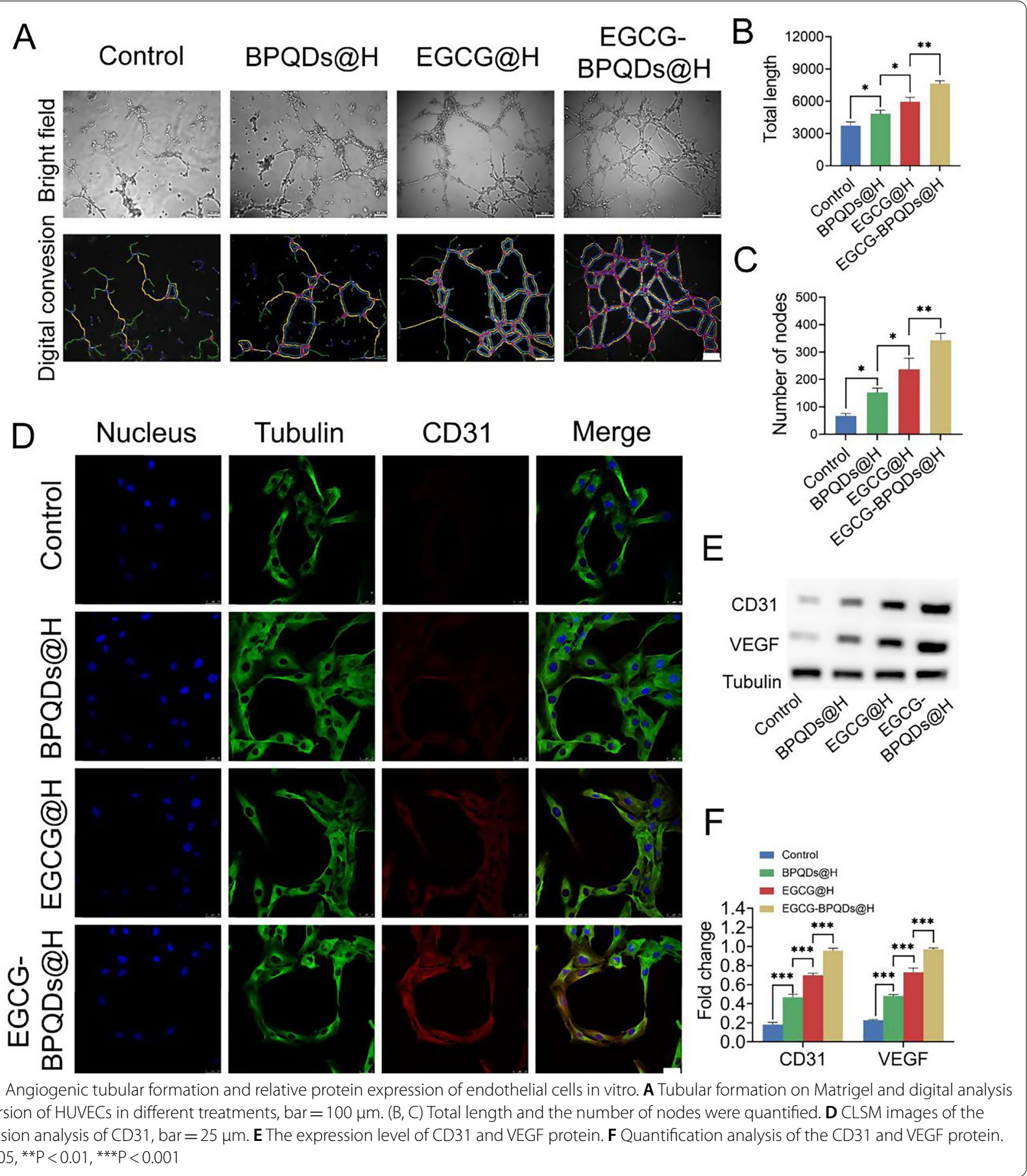

wound closure in the continued bacterial infected burnwound healing experiment (Fig. 7B). More specifically, the relative wound area on day 21 exhibited that local EGCG-BPQDs@H with NIR irradiation application leading to $92.4 \%$ wound closure, which was conspicuously higher than the control group (61.1\%) (Fig. 7C). Remarkably, the elevated temperature induced by the photothermal effect was detected upon NIR irradiation. The highest temperature of wound region treated with BPQDs@H or EGCG-BPQDs@H was $\sim 55^{\circ} \mathrm{C}$ after 5 min' irradiation, revealing prominent photothermal performance of black phosphorus quantum dots (Fig. 7D).

One major concern about photothermal therapy (PTT) is that local hyperpyrexia may cause secondary injury 


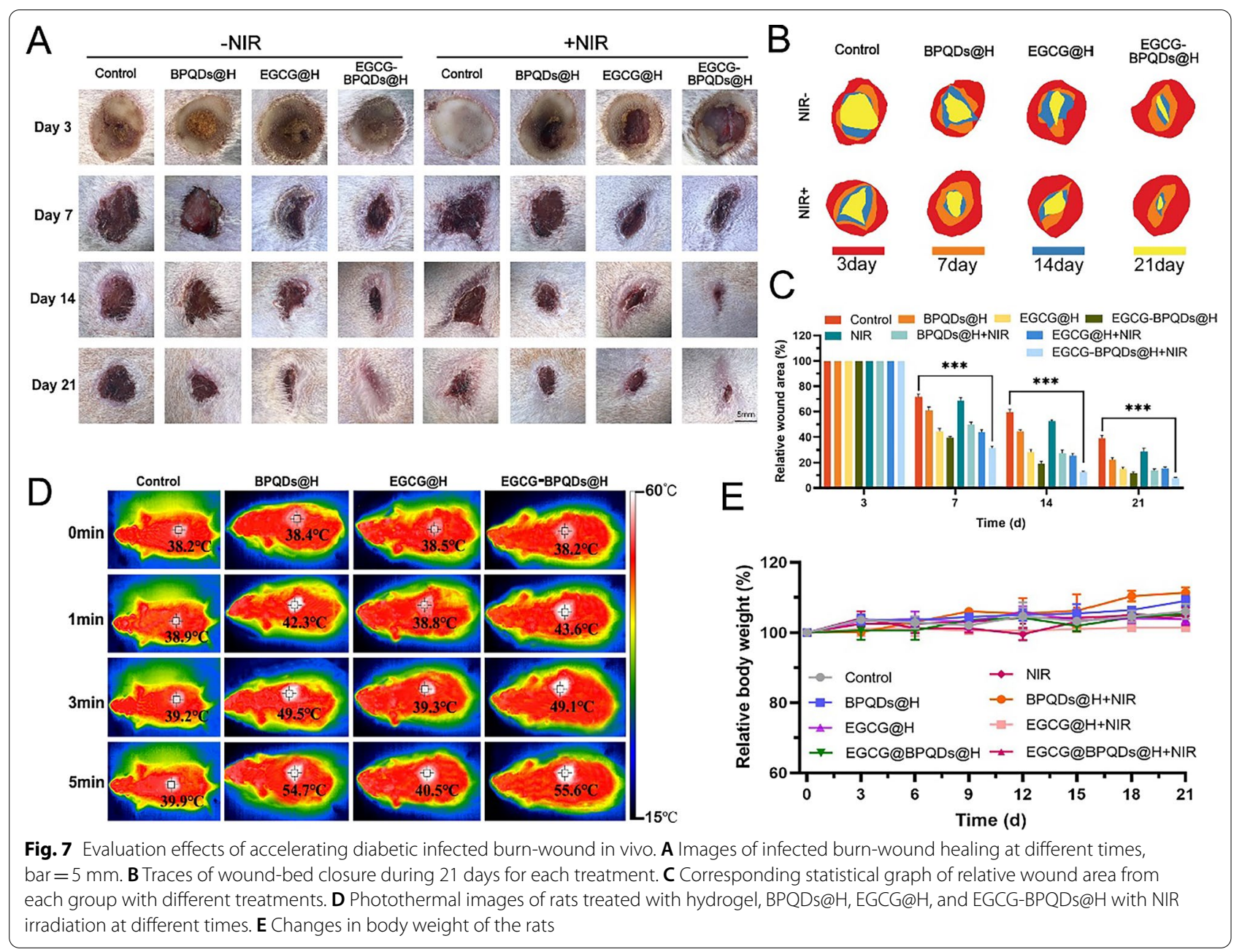

to the burn wound. The impairment to cells around the wound caused by PTT is far less serious than that caused by bacterial infection. This is acceptable for PTT-mediated improvement of blood flow and increased oxygen supply to promote burn-wound healing. In contrast, the trauma area in the control or EGCG@H groups showed temperature increase of only $1.7^{\circ} \mathrm{C}$ and $2.0{ }^{\circ} \mathrm{C}$. Moreover, no significant body weight fluctuations were observed in all groups during the treatment period (Fig. 7E), indicating the satisfactory bio-safety of these tested nanomaterials. In the meantime, an obvious healing effect can also be observed in the treatment of EGCG-BPQDs@H compared with other single treatment groups. While ECGC@ BPQDs@H+NIR group indicating the minimum wound area and fastest healing rate, which revealed the EGCGBPQDs@H could be used to apply the drug-resistant bacterial infected diabetic wound in vivo with assistance of the photothermal and photocatalytic effects. Next, to verify the in vivo antimicrobial activity, we collected MRSA from the wound skin and performed a bacterial colony count test using the LB agar plate smear method. As shown in Additional file 1: Fig. S6A, few colonies were seen in the EGCG-BPQDs@H+NIR group, and the results were consistent with in vitro experiments. For the clinical treatment of diabetic burn patients, this near-infrared light-responsive composite hydrogel can promote repair and resistance to drug-resistant bacterial infections. In addition, the superficial application and the ability to achieve rapid wound healing provide ease and feasibility for clinical application. Phototherapy has attracted a great deal of research due to its remote controllability, deep tissue penetration, and non-invasive nature [45-48]. Its potential for a wide range of biological applications includes, but is not limited to, trauma repair, tumor treatment [49-51], and probe imaging [52, 53]. As its antibacterial mechanism is different from that of antibiotics, the use of PTT can effectively avoid the development of resistance. These are very attractive for clinical applications. And by H\&E staining (Additional file 1: Fig. S6B), many inflammatory cells were found in 
the wounds treated with the control group. After treatment with EGCG-BPQDs@H with NIR, there was a significant reduction in the number of inflammatory cells in the wounds relative to the other groups.

To observe the histological changes in wound healing, $H \& E$ and Masson staining were performed to study the mechanism behind this process. Compared with other groups, control and BPQDs@H groups could not develop intact epidermal tissue. EGCG@H and EGCG-BPQDs@H groups had completed the procedure of re-epithelialization. The epithelium of the EGCGBPQDs@H with NIR group was smoother and more regulated than of the EGCG-BPQDs@H group, and follicles began to develop around the wound (Fig. 8A). Matson staining was then applied to samples from different groupings to reflect collagen deposition in the sarcomeres. As a result, only a few collagen fiber bundles were formed in the control and BPQDs@H groups. There was still large spaced and a loose reticular arrangement between the collagen fibers and skin tissue remaining. In contrast, EGCG-BPQDs@H group had an intact epidermal structure and dense, regular collagen fibers with a larger collagen deposition area at the wound (Fig. 8B). Subsequently, western blot analysis revealed that EGCG-modified BPQDs had increased the expression of key signaling molecules in the PI3K/AKT and ERK1/2pathways (Fig. 8C). Quantitative analysis revealed that phosphoinositide 3-kinase (PI3K), protein kinase $\mathrm{B}(\mathrm{AKT})$, and extracellular signal-regulated kinase $(E R K 1 / 2)$ were significantly increased in the EGCG-BPQDs@H group compared to the control group (Fig. 8D). These signaling pathways direct cells proliferation and differentiation, which was attributed to the stimulative effect of the phosphate/phosphonate ions following the degradation of BPQDs. Figure $8 \mathrm{E}$ summarizes the previous findings on how nanomaterials induce signaling pathways to direct cell proliferation and differentiation. In addition, we subcutaneously injected the hybrid hydrogel into the dorsal side of the rats (Additional file 1: Fig. S7). The experimental results revealed a gradual decrease in hydrogel content due to in vivo metabolism. During this process, there was no swelling or necrosis of the tissue around the hydrogel, indicating that the hydrogel system has favorable biocompatibility.

\section{Biological safety in vivo}

The results presented above fully attested to the prominent properties and potential of EGCG-BPQDs@H with NIR irradiation treatment in antibacterial activity and wound healing. Meanwhile, it is crucial to assess the biocompatibility of combined photothermal therapy in vivo for its practical application. Due to the characteristics of nanomaterials in direct contact with the burn-wound, blood biochemical analysis, and histological examination were applied to evaluate the biocompatibility of the cooperative treatment in vivo. BP has been widely used in drug delivery with high biocompatibility [54]. Additionally, biodegradation products of BP are phosphate ions and phosphonates, which are normally present in the blood [55]. Biochemistry analyses were carried out for blood at the twenty-first day. The liver function indexes (such as ALT and AST) and kidney function indexes (such as BUN and CREA) in the EGCGBPQDs@H+NIR system were in the normal range (Fig. 9A). Subsequently, for EGCG-BPQDs@H+NIR system, H\&E staining did not detect apparent inflammation and necrosis in the normal anatomical structure of various organs after treatment (Fig. 9B). In addition, we co-cultured the nanomaterial hydrogel with $\mathrm{HaCaTs}$ and found that the cells had good morphology with no obvious deformation or necrosis, proving their good biocompatibility (Additional file 1: Fig. S8). The hemolytic properties of the nanomaterial hydrogels were evaluated by measuring the hemolytic properties of red blood cells. The hemolysis rate of BPQDs@H, EGCG@H, and EGCGBPQDs@H was significantly lower (less than 5\%) after incubation of erythrocytes with each group of composites compared with water, indicating that the composite hydrogels had no significant hemolytic effect (Additional file 1: Fig. S9). These results strongly demonstrated that the EGCG-BPQDs@H with NIR irradiation system could be competent for a safe and effective therapeutic strategy to accelerate infected burn-wound healing.

\section{Conclusion}

In summary, the present work successfully constructed photocatalytic, photothermal effects of EGCG modified black phosphorus quantum dots as multifunctional nanoplatforms and evaluated their antibacterial ability and wound healing activity in MRSA-infected wounds under diabetic conditions. The resulted EGCG-BPQDs present an effective sterilization rate of $88.6 \%$ for MRSA. Combining the photothermal effect of our sterilization depot promotes the release of EGCG, and EGCG-BPQDs@H could produce ${ }^{1} \mathrm{O}_{2}$ in the case of NIR light. Molecular biology analysis demonstrated that EGCG-BPQDs significantly upregulated CD31 nearly fourfold and basic fibroblast growth factor (bFGF) nearly twofold, which were beneficial for promoting the proliferation of vascular endothelial cells and skin epidermal cells. Under NIR irradiation, EGCG-BPQDs hydrogel (EGCG-BPQDs@H) treated MIDBW area could rapidly raise temperature up to $55{ }^{\circ} \mathrm{C}$ for sterilization. The MIBDW closure rate of rats after 21 days of treatment was $92.4 \%$, much better than $61.1 \%$ of the control group. The engineered EGCG-BPQDs were found to promote MIDBW healing 


\section{A Control \\ BPQDs@H \\ EGCG@H \\ BPQDs@H}
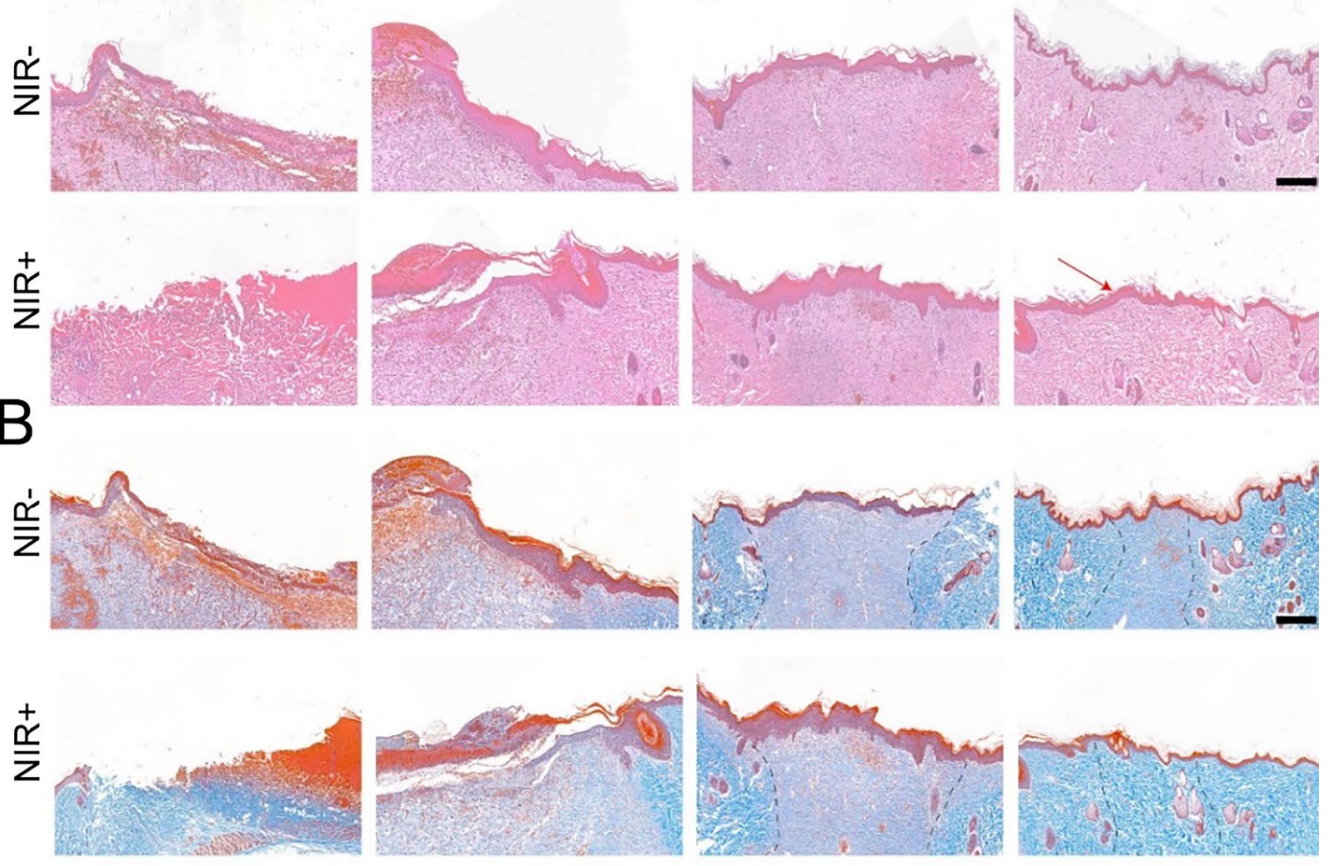

C

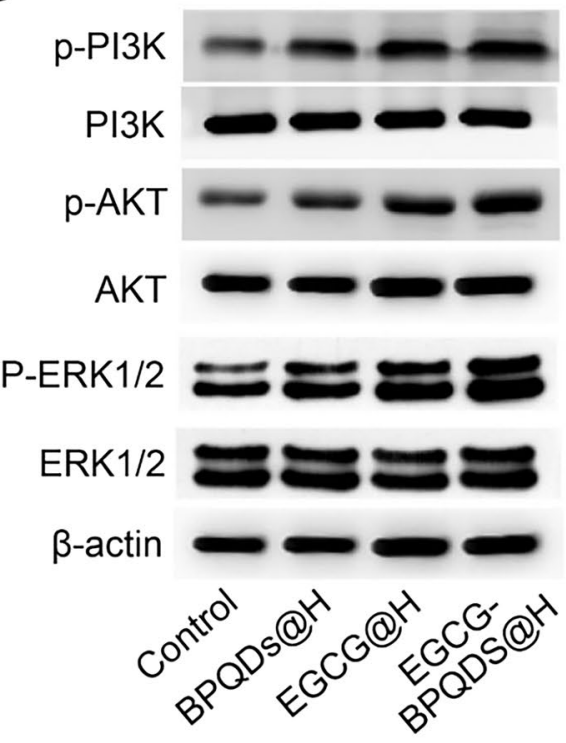

D

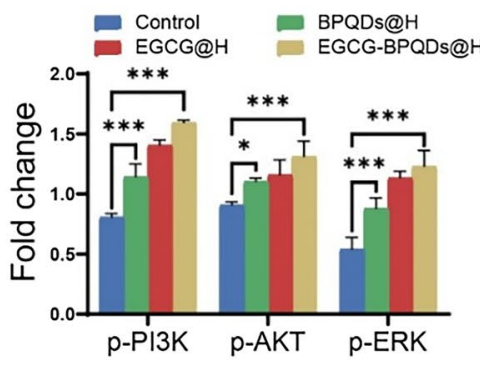

$E$

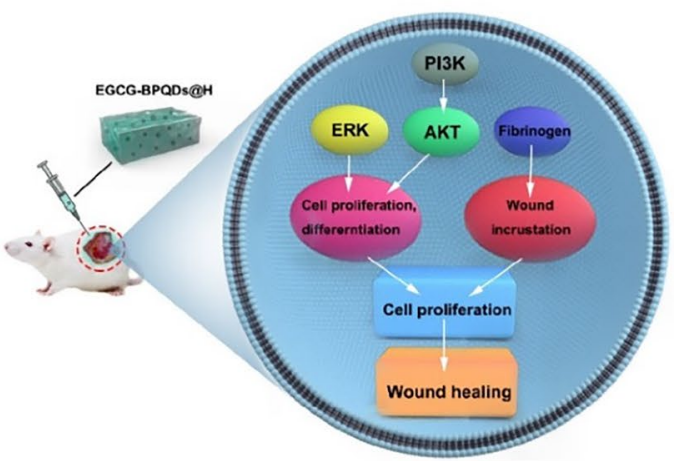

Fig. 8 Evaluation on the healing-promoting effect of the nanoplatforms on infected burns rats. A H\&E staining of wound sites with different treatments, the red arrow indicates intact epidermis, bar $=200 \mu \mathrm{m}$. B Masson staining of the wound tissues, dotted line indicates collagen at the wound, bar $=200 \mu \mathrm{m}$. CWestern blot analysis. D Quantification for the molecules involved in the signaling pathways for burn wound healing. E Scheme diagram showing nanomaterials direct cell proliferation and enhanced fibrinogen expression to accelerate wound healing 


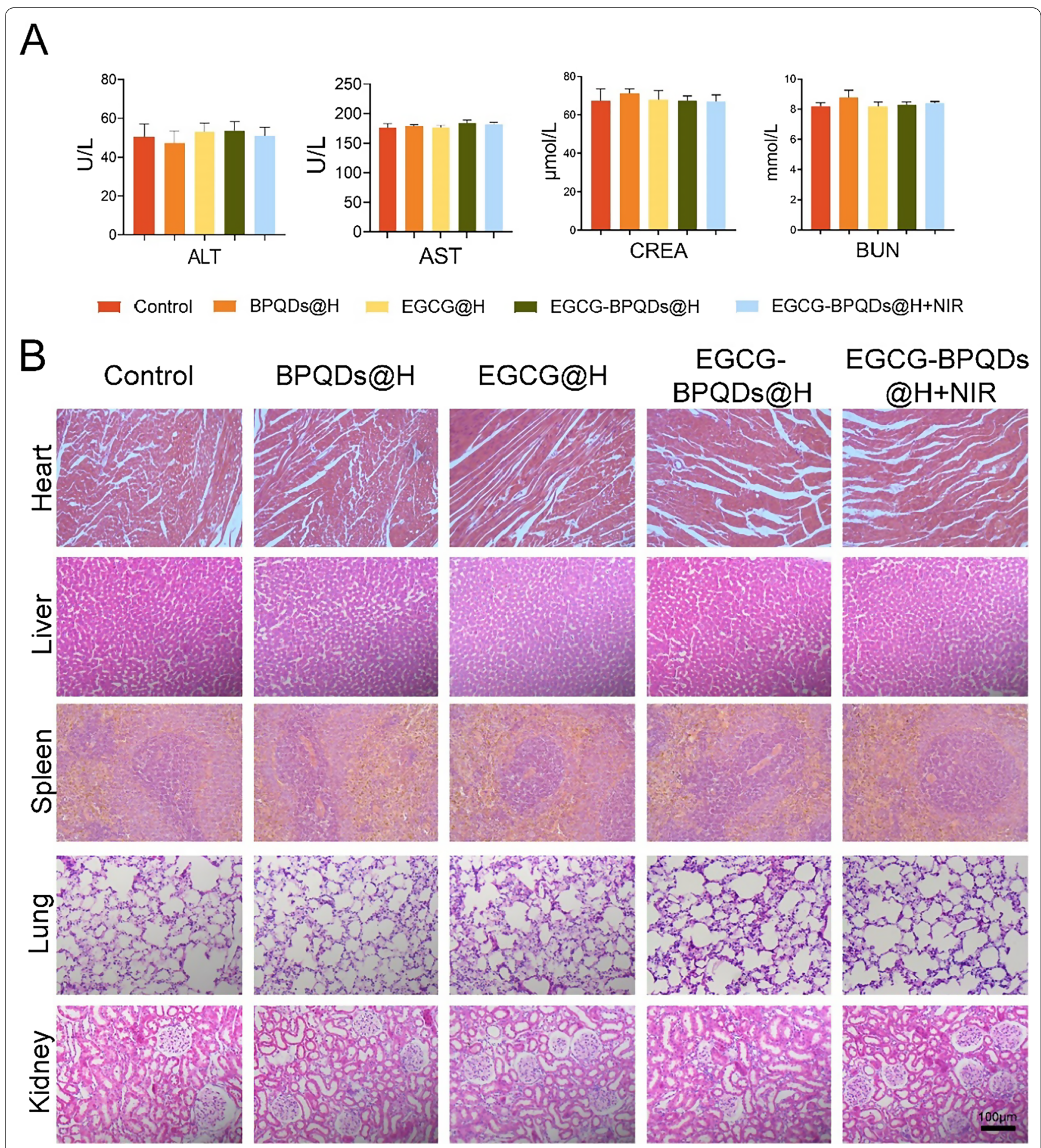

Fig. 9 Preliminary toxicity study in vivo. A Hepatorenal function test for ALT, AST, CREA and BUN. B Histological toxicological observation of H\&E staining of the heart, liver, spleen, lung, and kidney with different treatments, bar $=100 \mu \mathrm{m}$

by triggering the PI3K/AKT and ERK1/2 signaling pathways, enhancing cell proliferation and differentiation. In addition, the intravenous circulation experiment showed good biocompatibility of EGCG-BPQDs. No obvious damage to major rat organs was observed. The obtained results demonstrate that EGCG-BPQDs promise multifunctional nanoplatforms for MIDBW healing. The treatment of acute and chronic difficult-to-heal skin wounds 
associated with infection has been an important clinical issue. For the clinical treatment of diabetic burn patients, this NIR light-responsive composite hydrogel can promote repair and resistance to drug-resistant bacterial infections. In addition to the superficial application and the ability to achieve rapid wound healing also provide convenience and feasibility in clinical applications. Thus, the results of this study provide strong evidence for the clinical applicability of EGCG-BPQDs@H for the successful treatment of wounds in complex disease conditions such as diabetes with infected burn wounds. This type of hydrogel dressing is expected to provide clinical candidates in the future.

\section{Abbreviations}

MRSA: Methicillin-resistant Staphylococcus aureus; EGCG: Epigallocatechin gallate; BPQDs: Black phosphorus quantum dots; NIR: Near-infrared; bFGF: Basic fibroblast growth factor; BP: Black phosphorus; ${ }^{1} \mathrm{O}_{2}$ : Singlet oxygen; $E$. coli: Gram-negative bacteria Escherichia coli; DMEM: Dulbecco's modified Eagle's medium; MTT: 3-(4,5-Dimethylthiazol-2-yl)-2,5-diphenyl tetrazolium bromide; FBS: Fetal bovine serum; HaCat: Human skin keratinocytes cells; HUVEC: Human umbilical vein endothelial cells; ABDA: The 2,2'-bis(anthracene9,10-diylbis(methylene))-dimalonic acid; NMP: 1-Methyl-2-pyrrolidone; DLS: Dynamic laser scattering; TEM: Transmission electron microscope; CLSM: Confocal laser scanning microscopy; STZ: Streptozotocin; SEM: Scanning electron microscope; DCFH-DA: $2^{\prime}, 7^{\prime}$-Dichlorodihydrofluorescein diacetate; DAPI: 4',6-Diamidino-2-phenylindole solution; DMSO: Dimethyl sulfoxide; ALT: Alanine aminotransferase; AST: Aspartate aminotransferase; CREA: Creatinine.
\end{abstract}

\section{Supplementary Information}

The online version contains supplementary material available at https://doi. org/10.1186/s12951-021-01106-w.

Additional file 1. Additional figures.

\section{Acknowledgements}

Not applicable.

\section{Authors' contributions}

SBX, LNC, SCH: conception and design of the experiment, as well as manuscript writing; YNH, XJZ, SBX: collection an assembly of data and data interpretation; SBX, SCH: perform the experiment and edit manuscript. ZHC, XLR, XFM: conception and design, financial support and the preparation. All authors read and approved the final manuscript.

\section{Funding}

This work was supported by National Natural Science Foundation of China (Nos. 82072076, 81771987, and 82072165), and Young Top Talents in Xing Liao Talents Program of Liaoning Province (XL YC1807183).

\section{Availability of data and materials}

The datasets used and/or analyzed during the current study are available from the corresponding author on reasonable request.

\section{Declarations}

\section{Ethics approval and consent to participate}

Animal care conformed to institutional guidelines. All animal studies were agreed by the Institutional Ethics Committee of Jinzhou Medical University.

\section{Consent for publication}

All authors agree to be published.

\section{Competing interests}

No potential conflicts of interest were disclosed.

Received: 13 October 2021 Accepted: 26 October 2021

Published online: 10 November 2021

\section{References}

1. B Cao F Xiao D Xing 2018 Polyprodrug antimicrobials: remarkable membrane damage and concurrent drug release to combat antibiotic resistance of methicillin-resistant Staphylococcus aureus Small 14411802008

2. J Yang X Zhang C Liu 2020 Biologically modified nanoparticles as theranostic bionanomaterials Progr Mater Sci 1100768

3. S Chen S Zhang M Galluzzi 2019 Insight into multifunctional polyester fabrics finished by one-step eco-friendly strategy Chem Eng J 358634 642

4. S Hussain J Joo J Kang 2018 Antibiotic-loaded nanoparticles targeted to the site of infection enhance antibacterial efficacy Nat Biomed Eng 2295 103

5. CN Dang YDM Prasad AJM Boulton 2003 Methicillin-resistant Staphylococcus aureus in the diabetic foot clinic: a worsening problem Diabet Med 202159161

6. E Katsoulis I Bissell DG Hargreaves 2006 MRSA pyogenic flexor tenosynovitis leading to digital ischaemic necrosis and amputation Journal of Hand Surgery 313350352

7. MA Farha S French JM Stokes 2017 Bicarbonate alters bacterial susceptibility to antibiotics by targeting the proton motive force ACS Infect Dis 4 3382390

8. Z Lei W Zhu X Zhang 2021 Bio-Inspired Ionic Skin for Theranostics Adv Func Mater 3182008020

9. X Li H Wang H Chen 2020 Dynamic covalent synthesis of crystalline porous graphitic frameworks Chem 64933944

10. Y Wang Y Yu J Guo 2020 Bio-inspired stretchable, adhesive, and conductive structural color film for visually flexible electronics Adv Func Mater 30 322000151

11. Z Xu Z Qiu Q Liu 2018 Converting organosulfur compounds to inorganic polysulfides against resistant bacterial infections Nat Commun 91113

12. X Zhang G Chen Y Yu 2020 Bioinspired adhesive and antibacterial microneedles for versatile transdermal drug delivery Research 2020 3672120

13. Y Xiang C Mao X Liu 2019 Rapid and superior bacteria killing of carbon quantum dots/ZnO decorated injectable folic acid-conjugated PDA hydrogel through dual-light triggered ROS and membrane permeability Small 15221900322

14. A Krizsan D Volke S Weinert 2014 Insect-derived proline-rich antimicrobial peptides kill bacteria by inhibiting bacterial protein translation at the 70 S ribosome Angew Chem Int Ed 53451223612239

15. K Xie Z Zhou Y Guo 2019 Long-term prevention of bacterial infection and enhanced osteoinductivity of a hybrid coating with selective silver toxicity Adv Healthcare Mater 851801465

16. Z Li Q Fu J Ye 2020 Ag+-coupled black phosphorus vesicles with emerging NIR-II photoacoustic imaging performance for cancer immunedynamic therapy and fast wound healing Angew Chem Int Ed 5949 2220222209

17. X Zhang G Chen Y Liu 2020 Black phosphorus-loaded separable microneedles as responsive oxygen delivery carriers for wound healing ACS Nano 14559015908

18. L Xu Y Zhang H Pan 2020 Preparation and performance of radiata-pinederived polyvinyl alcohol/carbon quantum dots fluorescent films Materials 13167

19. J Ouyang X Ji X Zhang 2020 In situ sprayed NIR-responsive, analgesic black phosphorus-based gel for diabetic ulcer treatment Proc Natl Acad Sci 117462866728677

20. B Huang L Tan X Liu 2019 A facile fabrication of novel stuff with antibacterial property and osteogenic promotion utilizing red phosphorus and near-infrared light Bioactive Mater 41721

21. J LiX Liu L Tan 2019 Zinc-doped Prussian blue enhances photothermal clearance of Staphylococcus aureus and promotes tissue repair in infected wounds Nat Commun 101115 
22. H Mao J Xu Y Hu 2015 The effect of high external pressure on the structure and stability of MOF a-Mg 3 (HCOO) 6 probed by in situ Raman and FT-IR spectroscopy J Mater Chem A 3221197611984

23. Z Chen CWang J Chen 2013 Biocompatible, functional spheres based on oxidative coupling assembly of green tea polyphenols J Am Chem Soc 1351141794182

24. G Chen L He P Zhang 2020 Encapsulation of green tea polyphenol nanospheres in PVA/alginate hydrogel for promoting wound healing of diabetic rats by regulating PI3K/AKT pathway Mater Sci Eng C 110110686

25. X Zhang G Parekh B Guo 2019 Polyphenol and self-assembly: metal polyphenol nanonetwork for drug delivery and pharmaceutical applications Fut Drug Discov 11 FDD7

26. ZX Tea C Prevention 2015 Tea and Cancer Prevention J Cancer Res Updates 426573

27. P Zhang L He J Zhang 2020 Preparation of novel berberine nano-colloids for improving wound healing of diabetic rats by acting Sirt1/NF-KB pathway Colloids Surf B 187110647

28. M Batmunkh M Myekhlai ASR Bati 2019 Microwave-assisted synthesis of black phosphorus quantum dots: efficient electrocatalyst for oxygen evolution reaction J Mater Chem A 7211297412978

29. Q Gao X Zhang W Yin 2018 Functionalized MoS2 nanovehicle with nearinfrared laser-mediated nitric oxide release and photothermal activities for advanced bacteria-infected wound therapy Small 14451802290

30. M Akagawa T Shigemitsu K Suyama 2003 Production of hydrogen peroxide by polyphenols and polyphenol-rich beverages under quasiphysiological conditions Biosci Biotechnol Biochem 671226322640

31. SS Lucky N Muhammad Idris Z Li 2015 Titania coated upconversion nanoparticles for near-infrared light triggered photodynamic therapy ACS Nano 91191205

32. Y Hu S Huang X Zhao 2021 Preparation of Photothermal Responsive and ROS generative Gold Nanocages for Cancer Therapy Chem Eng J 1 129744

33. Z Xiong X Zhang S Zhang 2018 Bacterial toxicity of exfoliated black phosphorus nanosheets Ecotoxicol Environ Saf 161507514

34. L Chang S Huang X Zhao 2021 Preparation of ROS active and photothermal responsive hydroxyapatite nanoplatforms for anticancer therapy Mater Sci Eng C 125112098

35. R Zhao W Kong M Sun 2018 Highly stable graphene-based nanocomposite (GO-PEI-Ag) with broad-spectrum, long-term antimicrobial activity and antibiofilm effects ACS Appl Mater Interfaces 10211761717629

36. Y Zhao X Dai X Wei 2018 Near-infrared light-activated thermosensitive liposomes as efficient agents for photothermal and antibiotic synergistic therapy of bacterial biofilm ACS Appl Mater Interfaces 10171442614437

37. KJ Yin K Olsen M Hamblin 2012 Vascular endothelial cell-specific microRNA-15a inhibits angiogenesis in hindlimb ischemia J Biol Chem 287322705527064

38. Z Chen J Duan Y Diao 2021 ROS-responsive capsules engineered from EGCG-Zinc networks improve therapeutic angiogenesis in mouse limb ischemia Bioactive Materials 61111

39. Z Xu J Li X Zhou 2019 The combined use of tea polyphenols and lactobacillus plantarum ST8SH bacteriocin in a rabbit model of infection following femoral fracture with internal fixation Med Sci Monit 25312 317

40. X Gao Z Xu G Liu 2021 Polyphenols as a versatile component in tissue engineering Acta Biomater 1195774

41. J Zhu M Zhang Y Gao 2020 Tetrahedral framework nucleic acids promote scarless healing of cutaneous wounds via the AKT-signaling pathway Signal Transduct Target Ther 51111

42. Tonnesen M G, Feng X, Clark R A F. Angiogenesis in wound healing. Journal of Investigative Dermatology Symposium Proceedings. Elsevier, 2000, 5(1): 40-46

43. MN Nakatsu RCA Sainson JN Aoto 2003 Angiogenic sprouting and capillary lumen formation modeled by human umbilical vein endothelial cells (HUVEC) in fibrin gels: the role of fibroblasts and Angiopoietin-1 Microvasc Res 662102112

44. CWang C Liang R Wang 2020 The fabrication of a highly efficient selfhealing hydrogel from natural biopolymers loaded with exosomes for the synergistic promotion of severe wound healing Biomater Sci 81313324

45. N Kong H Zhang C Feng 2021 Arsenene-mediated multiple independently targeted reactive oxygen species burst for cancer therapy Nat Commun 121118

46. X Ji L Ge C Liu 2021 Capturing functional two-dimensional nanosheets from sandwich-structure vermiculite for cancer theranostics Nat Commun 121117

47. C Liu S Sun Q Feng 2021 Arsenene nanodots with selective killing effects and their low-dose combination with B-elemene for cancer therapy Adv Mater 12102054

48. WY Kim M Won S Koo 2021 Mitochondrial H2Sn-mediated anti-inflammatory theranostics Nano-Micro Letters 131113

49. D Gao T Chen S Chen 2021 Targeting hypoxic tumors with hybrid nanobullets for oxygen-independent synergistic photothermal and thermodynamic therapy Nano-Micro Letters 131121

50. Y Wang D Gao Y Liu 2021 Immunogenic-cell-killing and immunosuppression-inhibiting nanomedicine Bioactive Mater 6615131527

51. D Gao X Guo X Zhang 2020 Multifunctional phototheranostic nanomedicine for cancer imaging and treatment Materials Today Bio 5100035

52. $Y$ Zheng H Wei $P$ Liang 2021 Near-Infrared-Excited Multicolor Afterglow in Carbon Dots-Based Room-Temperature Afterglow Materials Angew Chem Int Ed 60412225322259

53. G Parekh Y Shi J Zheng 2018 Nano-carriers for targeted delivery and biomedical imaging enhancement Ther Deliv 96451468

54. JR Choi KW Yong JY Choi 2018 Black phosphorus and its biomedical applications Theranostics 8410051026

55. J Shao H Xie H Huang 2016 Biodegradable black phosphorus-based nanospheres for in vivo photothermal cancer therapy Nat Commun 711 13

\section{Publisher's Note}

Springer Nature remains neutral with regard to jurisdictional claims in published maps and institutional affiliations.

\footnotetext{
Ready to submit your research? Choose BMC and benefit from:

- fast, convenient online submission

- thorough peer review by experienced researchers in your field

- rapid publication on acceptance

- support for research data, including large and complex data types

- gold Open Access which fosters wider collaboration and increased citations

- maximum visibility for your research: over $100 \mathrm{M}$ website views per year
}

At BMC, research is always in progress.

Learn more biomedcentral.com/submissions 